\title{
Whipworm and roundworm infections
}

Kathryn J. Else ${ }^{1 凶}$, Jennifer Keiser ${ }^{2,3}$, Celia V. Holland4, Richard K. Grencis ${ }^{1}$, David B. Sattelle, Ricardo T. Fujiwara ${ }^{6}$, Lilian L. Bueno ${ }^{6}$, Samuel O. Asaolu', Oluyomi A. Sowemimo7 and Philip J. Cooper ${ }^{8,9}$

Abstract | Trichuriasis and ascariasis are neglected tropical diseases caused by the gastrointestinal dwelling nematodes Trichuris trichiura (a whipworm) and Ascaris lumbricoides (a roundworm), respectively. Both parasites are staggeringly prevalent, particularly in tropical and subtropical areas, and are associated with substantial morbidity. Infection is initiated by ingestion of infective eggs, which hatch in the intestine. Thereafter, T. trichiura larvae moult within intestinal epithelial cells, with adult worms embedded in a partially intracellular niche in the large intestine, whereas A. lumbricoides larvae penetrate the gut mucosa and migrate through the liver and lungs before returning to the lumen of the small intestine, where adult worms dwell. Both species elicit type 2 anti-parasite immunity. Diagnosis is typically based on clinical presentation (gastrointestinal symptoms and inflammation) and the detection of eggs or parasite DNA in the faeces. Prevention and treatment strategies rely on periodic mass drug administration (generally with albendazole or mebendazole) to at-risk populations and improvements in water, sanitation and hygiene. The effectiveness of drug treatment is very high for A. lumbricoides infections, whereas cure rates for T. trichiura infections are low. Novel anthelminthic drugs are needed, together with vaccine development and tools for diagnosis and assessment of parasite control in the field.

Whipworms are large-intestinal nematode parasites of mammals. The scientific name for whipworms is Trichuris (which means 'hair tail'), a name applied by Johann Georg Roederer in 1761, who mistook the thin front end for the tail. Over 70 Trichuris spp. are recognized, including the medically important human parasite Trichuris trichiura (the aetiological agent of trichuriasis) and the pig whipworm Trichuris suis. Whipworms have been associated with humans for over 8,000 years, as evidenced by the presence of $T$. trichiura eggs in coprolites (fossilized faeces) found in both Old World and New World archaeological sites ${ }^{1-3}$. Roundworms (Ascaris spp.) are also intestinal nematodes, but, unlike whipworms, they dwell in the small intestine. Ascaris lumbricoides (first described by Carl Linnaeus in 1758) is the causative agent of the human disease ascariasis. In contrast to whipworms, the genus Ascaris differs from the genus Trichuris in that only one other Ascaris species has been described - Ascaris suum, a ubiquitous pathogen of pigs. After considerable debate as to whether these two ascarids are in fact distinct species, the current opinion is that they are two species, closely related at the phylogenetic level but reproductively isolated (that is, they are unable to interbreed successfully $)^{4}$. Like T. trichiura, A. lumbricoides has a long association with its human host, with eggs detected in embalming material from over 7,000 years ago ${ }^{5}$ (FIG. 1).
Both T. trichiura and A. lumbricoides are highly prevalent helminths (common name for parasitic worms) ${ }^{6,7}$. The infections occur by ingestion of embryonated (containing an embryo) eggs through contaminated soil or food. Both parasites contribute to chronic, longterm nutritional morbidity and affect cognitive development, although there is less evidence supporting this latter effect. Acute complications associated with A. lumbricoides infections of heavy intensity (that is, with a high worm burden) are intestinal obstruction and biliary ascariasis, whereas complications of T. trichiura infections include Trichuris dysentery syndrome (TDS) and rectal prolapse. The main approach to infection control is large-scale provision of anthelminthic treatment to children and girls and women of reproductive age, with accompanying improvements in access to clean water and sanitation, to reduce worm burden-associated morbidity $^{8}$. Whilst largely effective against ascariasis, mass drug administration (MDA) programmes have been substantially less so against trichuriasis, particularly in sub-Saharan Africa9.

In this Primer, we provide a current view of both T. trichiura and A. lumbricoides infections epidemiology, disease mechanisms, diagnosis, screening and prevention. We also review current management strategies and consider key research areas that may lead to improved control of these two important neglected tropical 


\author{
Author addresses \\ ${ }^{1}$ Lydia Becker Institute for Immunology and Inflammation, Faculty of Biology, Medicine \\ and Health, University of Manchester, Manchester Academic Health Science Centre, \\ Manchester, UK. \\ ${ }^{2}$ Department of Medical Parasitology and Infection Biology, Swiss Tropical and Public \\ Health Institute, Basel, Switzerland. \\ 'University of Basel, Basel, Switzerland. \\ ${ }^{4}$ Department of Zoology, School of Natural Sciences, Trinity College Dublin, Dublin, Ireland. \\ ${ }^{5}$ Centre for Respiratory Biology, UCL Respiratory, Rayne Building, University College \\ London, London, UK. \\ ${ }^{6}$ Department of Parasitology, Institute of Biological Sciences (ICB), Universidade Federal \\ de Minas Gerais, Belo Horizonte, Brazil. \\ 'Department of Zoology, Obafemi Awolowo University, Ile-Ife, Osun State, Nigeria. \\ ${ }^{8}$ Institute of Infection and Immunity, St George's University of London, London, UK. \\ ${ }^{9}$ Facultad de Ciencias Medicas, de la Salud y la Vida, Universidad Internacional del \\ Ecuador, Quito, Ecuador.
}

diseases. Further, we compare and contrast Trichuris spp. and Ascaris spp. infections, which, despite the parasites sharing several traits, differ in important areas, with relevant consequences for control strategies.

\section{Epidemiology}

T. trichiura and A. lumbricoides infections are highly prevalent worldwide, with estimates of 465 and 819 million affected humans, respectively, in $2010\left(\mathrm{REFS}^{6,10}\right)$. Owing to infection control efforts, the overall prevalence of ascariasis was estimated to decline by $10 \%$ between 2005 and 2015, whereas trichuriasis prevalence declined by only $2 \%{ }^{10}$. Although the oral-faecal route of infection is the same for both parasites, their geographical distributions do not perfectly overlap (FIG. 2), perhaps owing to spatial factors such as temperature, humidity and soil type; however, it is not known why the distributions diverge in some specific areas. In the endemic areas where the distributions overlap, co-infections frequently occur and probably result in exacerbation of morbidity and heavy infection intensities ${ }^{11-15}$. Co-infections often affect children and are generally underdiagnosed, as they are associated with non-specific gastrointestinal symptoms. Morbidity is most likely to occur among children with moderate to heavy infection intensities and is attributed to chronic effects on nutrition and growth. There are limited data to quantify the frequency of complications of trichuriasis and ascariasis, but, in 2017, the estimated number of deaths worldwide attributable to ascariasis was 3,205 , whereas no deaths were considered attributable to trichuriasis ${ }^{16}$.

\section{Trichuris trichiura}

T. trichiura infections are most frequent in warm and moist conditions in tropical and sub-tropical regions. Although zoonotic infections with other Trichiura spp. such as T. suis (from pigs) and Trichiura vulpis (from dogs) have been reported in humans, these parasites generally cause attenuated infections and rarely develop to sexual maturity in humans. Geographical information system tools that enable prediction of regions that are permissive for transmission, on the basis of spatial information on temperature, humidity and population density, have been used to estimate the geographical distribution of T. trichiura (FIG. 2). Transmission requires embryonation of $T$. trichiura eggs in the environment, and whilst eggs can survive temperatures below freezing, they will not embryonate in freezing conditions or if temperatures exceed $37^{\circ} \mathrm{C}\left(\mathrm{REFS}^{17,18}\right)$.

Under experimental conditions, humans can become infected with the pig whipworm $T$. suis ${ }^{17}$. These infections seem to only establish temporarily ${ }^{19}$, although one study ${ }^{20}$ found the maturation of $T$. suis to fecund, fully grown adult worms in a volunteer. Similarly, T. trichiura can be established in pigs, but the parasites do not persist in this host $^{17}$. Further, data indicate that the taxonomic, population and phylogenetic structure of T. trichiura is com$\mathrm{plex}^{21}$. Altogether, these data suggest that T. trichiura is not a single multi-host species but a series of lineages, some of which can infect multiple mammalian host species.

Prevalence. Human trichuriasis is a classic disease of poverty, in which a lack of education and access to sanitation and clean water within an ecologically permissive environment favours infection transmission. In such environments, community prevalence of infections can be $>90 \%$, and infections can particularly affect children of 5-15 years of age, who have the greatest parasite burdens ${ }^{22}$. Age-prevalence profiles are concave; prevalence peaks at an earlier age in areas of moreintense transmission than in areas where transmission is not as intense and probably relates to exposure risk (ingestion of eggs from a faecally contaminated environment). An age-dependent decline in prevalence is often observed in children of $>15$ years of age and in adults, probably owing to reduced exposure and possible age-acquired immunity.

Treatment of school-age children is considered a cost-effective strategy for the control of $T$. trichiura infection in communities in endemic areas: by cutting the number of infections in the primary parasite reservoir $^{23}$, transmission within communities is reduced. Temporal improvements in economic and environmental conditions, coupled with increased access to periodic chemotherapy for school-age children, have led to substantial declines in prevalence and intensity of infection in Asia since the year 2000, particularly in China and Indonesia ${ }^{6}$. Although similar declines have not been observed in Latin American and sub-Saharan African regions ${ }^{6,24}$, declines in the numbers of children with moderate to heavy infection intensities (the population most at risk of severe disease) have been observed in almost all settings in which school-age children have received repeated preventive chemotherapy ${ }^{25}$.

Risk factors. The risk of T. trichiura infection is not uniform within populations in endemic areas: a small proportion of infected individuals (typically $<10 \%$ in high-prevalence populations), generally children of 2-15 years of age, harbour most adult worms, whereas the remaining infected children and adults harbour few adult worms $\mathrm{s}^{23}$. Such aggregated distributions of adult worms (which may survive for 1-8 years in the human intestin ${ }^{26}$ ) within communities in endemic areas are typical of soil-transmitted helminths (STHs). There is evidence from some, but not all, epidemiological studies of an increased susceptibility to T. trichiura infection among some groups of individuals - for example, 
a

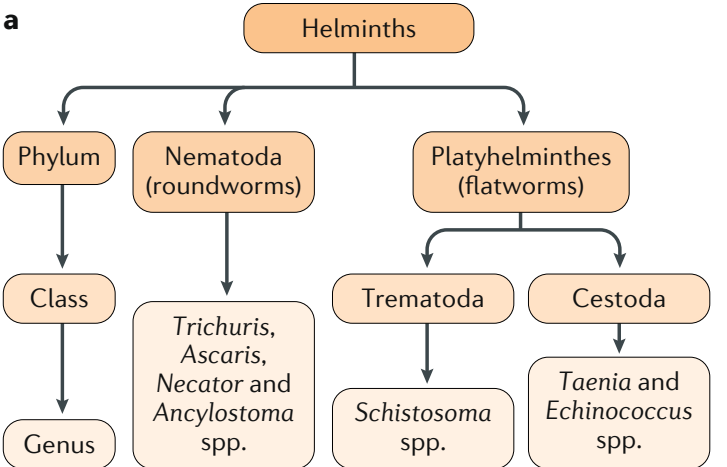

b

Trichuris trichiura and Ascaris lumbricoides

- Associated with humans for thousands of years

- Highly prevalent infections with an over-dispersed frequency distribution

- Life cycle in the human host starts with oral ingestion of embryonated

eggs and ends with shedding of unembryonated eggs

- Infection stimulates type 2 immunity

T. trichiura

- Adult worms are 3-5 cm long

- Adult worms live in the large intestine

- Pathology of the large intestine
A. lumbricoides

- Adult worms are 15-25 (male) and 20-35 (female) cm long

- Adult worms live in the small intestine

- Pathology of the liver, lungs

and small intestine

Fig. 1 | Soil-transmitted helminth infections. a | Helminth is an umbrella term for human multicellular endoparasites; most of these worms belong to the Nematoda and the Platyhelminthes phyla. A third phylum of parasitic worms exists: the Acanthocephala phylum; however, these worms very rarely infect humans, who are occasional accidental hosts. The Trematoda and Cestoda are classes of platyhelminths, whereas the so-called soil-transmitted helminths are found within the Nematoda. Examples of genera found within each phylum are included. $\mathbf{b}$ | Main similarities and differences between Trichuris trichiura and Ascaris lumbricoides parasites.

previously infected individuals are more likely to become reinfected after chemotherapy than uninfected individuals ${ }^{27}$. Individual susceptibility may be determined by one or more behavioural, environmental, genetic and immunological factors ${ }^{27}$. Further, individuals with heavy infection intensities tend to be those who re-acquire the highest parasite burdens following treatment ${ }^{18,27,28}$. T. trichiura clustered within families in rural China ${ }^{29}$, and a linkage analysis in Nepal identified two quantitative trait loci on chromosomes 9 and 18, respectively, that were associated with susceptibility to infection ${ }^{30}$, although the contributing genes at these loci remain unknown. Finally, a study in Brazil showed that susceptibility to T. trichiura infection was associated with polymorphisms in TGFB1 $\left(\mathrm{REF}^{31}\right)$.

\section{Ascaris lumbricoides}

Globally, A. lumbricoides was estimated to infect 819 million people in 2010 ( $\mathrm{REFS}^{6,32}$ ), with a similar geographical distribution in tropical and subtropical areas to that observed for trichuriasis (FIG. 2). Experimental and molecular evidence of possible cross-transmission indicates that humans can be infected by $A$. suum ${ }^{33-35}$, and pigs can harbour A. lumbricoides ${ }^{35}$. These data suggest that pigs might act as a potential reservoir of infection for humans and, more importantly, might point out a possible role of zoonotic infection by A. suum in humans ${ }^{36}$. The zoonotic potential of both $A$. suum and T. suis has been reviewed ${ }^{37}$.

Prevalence and risk factors. Ascariasis is also associated with poverty, and hence the lack of proper sanitary infrastructure and poor socio-economic conditions favour the transmission of the parasite ${ }^{38,39}$. Overall, an overdispersed frequency distribution ${ }^{40}$ is observed, with most individuals harbouring a low to moderate parasite burden and few hosts with heavy infection intensities. Socioeconomic factors, such as poor housing ${ }^{41}$ and deficiency in hygiene practices ${ }^{42}$, influence the intensity of infection. The infection intensities observed in adults are often lighter than those found in children ${ }^{43}$, and this observation might suggest a behaviour-mediated reduction of exposure or the development of acquired immunity with chronic exposure to the parasite. However, whereas experimental data in mice demonstrate a reduction in parasite burden after repeated exposure to A. suum ${ }^{44}$, the over-dispersed frequency distribution in humans is recorded in all age groups, indicating that neither age nor immunity are the primary determinants of variability in infection intensity.

Environmental and behavioural features ${ }^{45}$, as well as a host's genetics and immunity ${ }^{46-50}$, are important determinants of infection status ${ }^{51}$. Predisposition (that is, reinfection with similar or higher worm burdens than pretreatment burdens) is also an epidemiological phenomenon observed in human ascariasis ${ }^{43}$, as well as in trichuriasis. In a systematic review ${ }^{27}$, children were found to show greater predisposition to A. lumbricoides infection than adults, and girls were more predisposed than boys. Although the mechanisms that determine predisposition are not fully elucidated, exposure to infection and host susceptibility are likely to be important.

\section{Mechanisms/pathophysiology}

Studies on immunity to human whipworm and roundworm infections have generated interesting immune correlates with resistance to reinfection; however, it is through the use of animal models, and particularly the laboratory mouse, that understanding of pathological mechanisms has been gained. Novel imaging tools are beginning to provide unique insights into both host pathology and parasite behaviour ${ }^{52,53}$. Current knowledge on human infection, followed by insights from animal models, are discussed below, including, where possible, reflections on how findings in animal models fit with the human disease.

\section{Trichuris spp.}

The life cycles of all Trichuris spp. are similar (FIG. 3). After the ingestion of food or water contaminated with soil containing embryonated eggs, the eggs hatch in the large intestine (caecum and/or proximal colon); in the mouse, hatching of Trichuris muris eggs is triggered by the presence of bacteria, and probably similar 

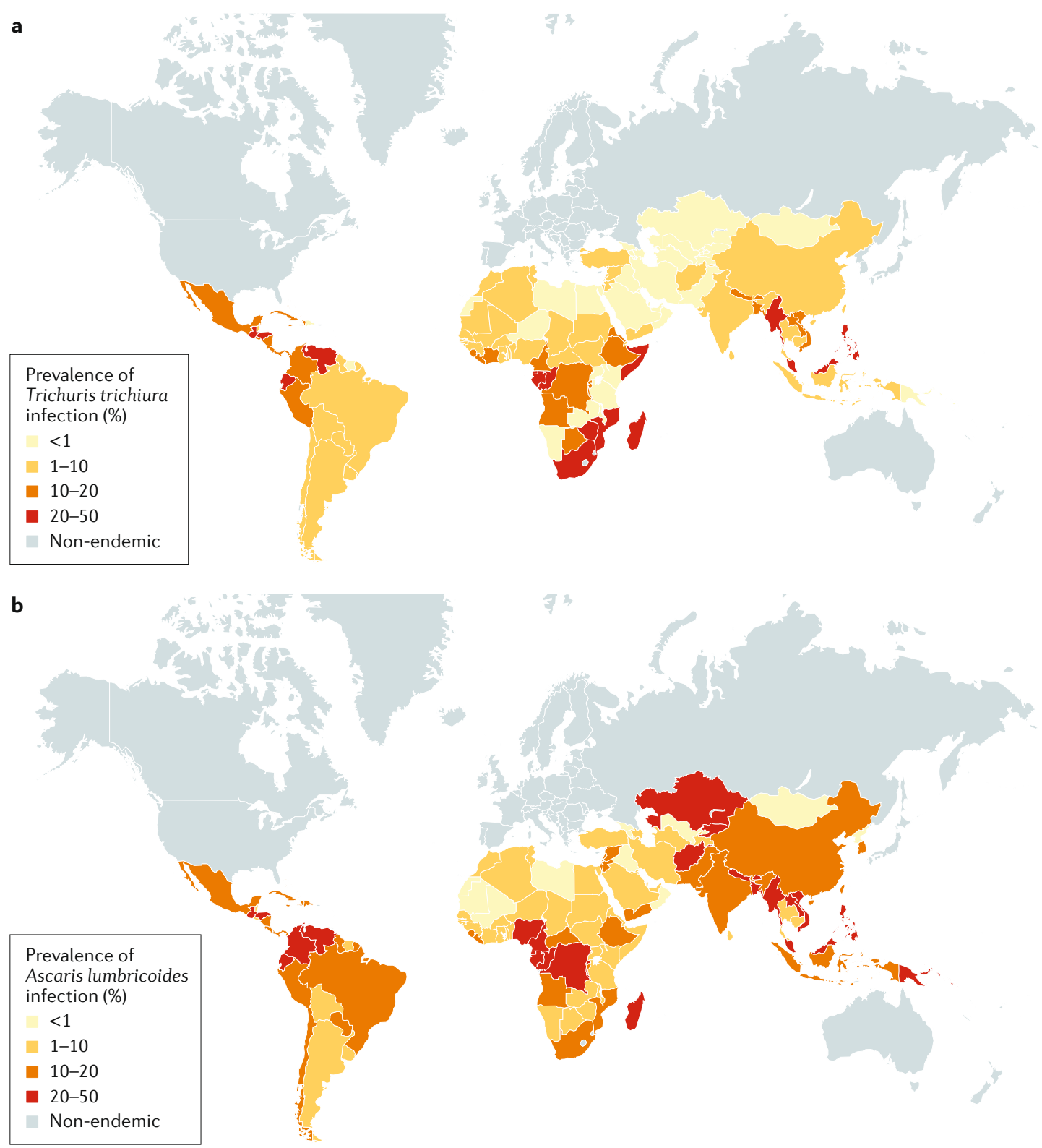

Fig. 2 | Prevalence of Trichuris trichiura and Ascaris lumbricoides infections in 2010. Distribution of Trichuris trichiura infection (part a) and Ascaris lumbricoides infection (part b), estimated on the basis of geostatistical models for sub-Saharan Africa and available empirical information for all other regions. T. trichiura infection may also occur in high-income regions, in populations living in conditions of poverty (such as aboriginal populations in Australia ${ }^{309}$ ) or among migrants ${ }^{7}$. In migrants, most infections are acquired elsewhere, as adequate hygiene and sanitation in most high-income regions provide limited opportunities for transmission. Adapted from REF. ${ }^{6}$, CC BY 2.0 (https://creativecommons.org/licenses/by/2.0/).

bacterial cues are applicable to egg hatching in other Trichuris spp. ${ }^{54}$. First stage (L1) larvae are released and penetrate the epithelial cells at the crypt base, where they create and inhabit an intracellular niche formed by a multicellular epithelial 'tunnel', the biology of which is unknown ${ }^{55}$. In this niche, L1 larvae grow and moult through the larval (L2, L3 and L4) and adult stages; timings of these moults are defined in the mouse model ${ }^{56}$, but the equivalent timings in humans are unclear. By the L3 stage, the parasite is no longer fully intracellular. Thus, its posterior end protrudes into the gut lumen, whilst its long thin anterior end, which contains the stichosome (a modified oesophagus comprising multiple cells (stichocytes) that duct into the oesophageal lumen), remains embedded within a syncytial tunnel of modified host epithelial cells, without substantially compromising the integrity of the gut barrier. The pre-patent period (that is, the time from infection to egg production) is 33-35 days in mice: adult male and female T. muris worms emerge around 32 days after infection, with fertilized adult females releasing 2,000 to 8,000 eggs per $\mathrm{day}^{57}$. Eggs of Trichuris spp. are expelled with host faeces unembryonated and, therefore, in a non-infective state. Embryonation takes 2-4 weeks, depending on environmental conditions ${ }^{17}$; by then, the L1 larva has developed within the egg and the egg is now infective. 
The life cycle of T. trichiura is similar to that of T. muris, although the timings of moults may differ. Thus, in humans, patent infections develop in 2-3 months, and adult worms, measuring $3-5 \mathrm{~cm}$, may survive for $1-8$ years in the human intestine ${ }^{26}$. Throughout their life cycle in the murine, porcine and human hosts, Trichuris spp. excrete and secrete a variety of parasite-derived molecules that interact with the host environment. Some molecules are antigenic and some are immunomodulatory ${ }^{58-60}$, but the functions of most are still to be determined. A better understanding of the host-parasite relationship is likely to support the development of new therapeutics (see Outlook).

Human trichuriasis: the evidence for type 2 acquired immunity to infection. Studying immunity to human trichuriasis is fraught with difficulty, with challenges including genetic heterogeneity, undefined infection history and exposure, and polyparasitism. Nevertheless, comprehensive cross-sectional serological field studies point clearly to a positive correlation between high antiT. trichiura IgE levels and decreasing infection intensity ${ }^{61}$, with IgE representing an antibody isotype controlled by type 2 immunity responses. There are no analyses of type 1 and type 2 cytokines released by peripheral blood leukocytes isolated from humans infected solely with T. trichiura and re-stimulated in vitro, as polyparasitism is usual in populations in endemic areas. However, data from populations infected with more than one species of gastrointestinal nematodes including T. trichiura strongly support the hypothesis that these infections induce type 2 immunity and regulatory responses ${ }^{62}$ and that acquired immunity requires type 2 protective immune responses that develop slowly after years, if not decades, of exposure ${ }^{63}$. Single-subject self-infection studies have contributed to our understanding of how

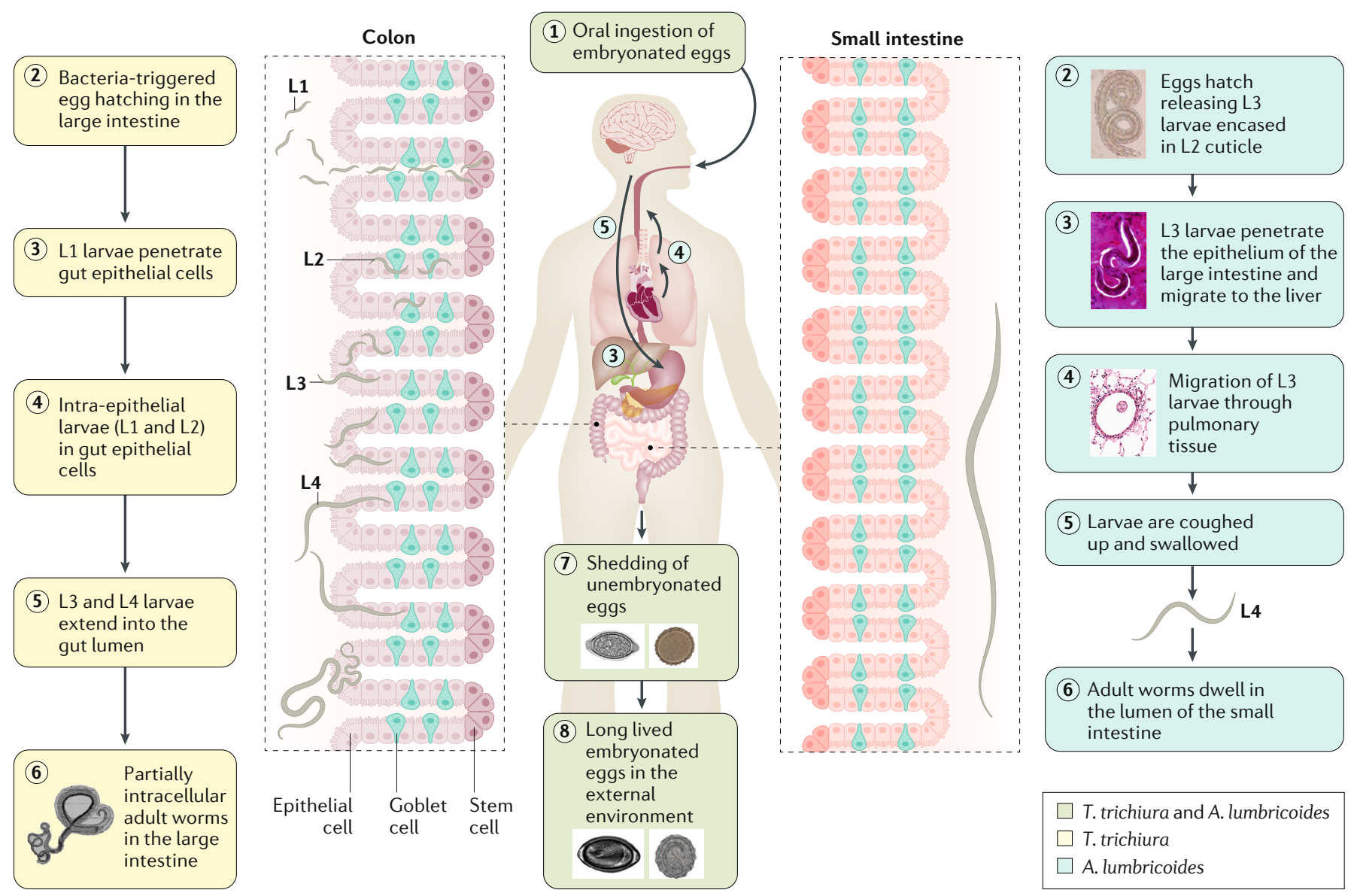

Fig. 3 | Life cycles of Trichuris trichiura and Ascaris lumbricoides. Both Trichuris trichiura and Ascaris lumbricoides infections are initiated by the oral ingestion of embryonated (infective) eggs. T. trichiura eggs hatch in the large intestine in response to molecular signals from bacteria. The first stage L1 larvae burrow into epithelial cells lining the crypts, and in this intracellular niche they grow and moult through to the adult stage. Thus, unlike A. lumbricoides, T. trichiura is an entirely enteric parasite. A. lumbricoides eggs hatch and release L3 larvae, covered by the L2 cuticle. Although the site of egg hatching has been a topic of some discussion, the current evidence points to the large intestine. L3 larvae penetrate the caecal and proximal colon mucosa and undergo a hepato-tracheal migration. L3 larvae first migrate to the liver, where the L2 cuticle is shed and further larval growth occurs.
Subsequently, larvae advance to the lungs, penetrate the alveolar spaces and move to the pharynx, from where they are coughed up and swallowed. In the small intestine, the now L4 larvae undergo a final moult (L5) and develop to adulthood. Sexually mature male and female T. trichiura and A. lumbricoides worms mate, and female worms produce unembryonated eggs that are shed in the faeces, where they develop to infectivity under appropriate conditions of temperature and moisture. Images of Ascaris suum larvae and larvae in lung (steps 2 and 4) courtesy of C. Holland. A. suum larva in liver (step 3) is adapted from REF. ${ }^{310}$, CC BY 4.0 (https://creativecommons.org/licenses/by/4.0/). Images of adult T. muris (step 6) and T. muris eggs (steps 7 and 8) courtesy of R. Forman, University of Manchester, UK. Images of A. lumbricoides eggs (steps 7 and 8) courtesy of G. Deslyper, Trinity College Dublin, Ireland. 
T. trichiura modulates human immunity: a longitudinal analysis of $\mathrm{T}$ cell subsets in mucosal biopsy samples and peripheral blood revealed a mixed mucosal $\mathrm{T}$ cell response (type $1 \mathrm{~T}$ helper $\left(\mathrm{T}_{\mathrm{H}} 1\right), \mathrm{T}_{\mathrm{H}} 2, \mathrm{~T}_{\mathrm{H}} 17$ and regulatory $\mathrm{T}\left(\mathrm{T}_{\mathrm{reg}}\right)$ cells), whilst circulating $\mathrm{T}$ helper cells became predominantly $\mathrm{T}_{\mathrm{H}} 2$ cells ${ }^{64}$. A second such study revealed an amelioration of the symptoms of colitis following T. trichiura infection, probably through improved $\mathrm{T}_{\mathrm{H}} 2$ cell-mediated and IL-22-mediated barrier function ${ }^{65}$.

Insights from animal models - type 2 immunity. Preclinical models have enabled us to delve more deeply into both the underlying cellular regulatory mechanisms that control resistance and susceptibility to infection and the effector mechanisms that eliminate the parasite. Although we focus on the T. muris mouse model of human trichuriasis, T. suis in pigs has also generated important data that reveal commonalities in type 2 immunity between mouse, human and pig ${ }^{66}$.

T. muris is the natural whipworm of mice and is genetically and antigenically similar to T. trichiura; these two species also show similar epidemiological patterns in their respective hosts. The importance of type 2 immunity in resistance to infection has been unequivocally demonstrated by many different research laboratories ${ }^{67-70}$, and research now focuses on untangling the contributions of cellular subsets ${ }^{68,71,72}$. An emerging concept is that the relevance of different cell types in promoting type 2 immunity is context dependent; thus, cellular contributions that are essential in one strain of mouse become redundant in a different strain or when the cytokine balance is artificially manipulated ${ }^{73,74}$, with important implications for translation of these findings to humans. One burning question is how protective type 2 responses develop (BOX 1); answers to this question might inform smart vaccine development in the future.

\section{Box 1 How do type 2 immune responses develop?}

Although several cell types (for example, innate lymphoid cells, B cells and macrophages) possess $\mathrm{MHC}$ II and can present antigens to $C D 4^{+} T$ cells (that is, naive $T$ helper $\left(T_{H}\right)$ cells that have not yet differentiated into a $\mathrm{T}_{\mathrm{H}}$ cell subtype), their in vivo contribution in murine trichuriasis is not fully defined. By contrast, dendritic cells (DCs) are potent antigen-presenting cells that play a key part in Trichuris muris infections in the mouse. Different subsets of DCs exist, with the IRF ${ }^{+} \mathrm{CD} 11 \mathrm{c}^{+} \mathrm{CD} 11 \mathrm{~b}{ }^{+} \mathrm{DC}$ s being the potent drivers of type 2 immunity after T. muris infection and IRF $8^{+} \mathrm{CD} 103^{+} \mathrm{DC}$ s being associated with type 1 immunity and, therefore, chronic infection ${ }^{314,315}$. How these subsets have compartmentalized roles is unclear, but mechanisms are probably mediated by both cell-intrinsic factors and external signals. For example, if the cellular phosphatidylinositol 3,4,5-trisphosphate 5-phosphatase 1 (also known as SHIP1) is deleted specifically from DCs, T. muris expulsion is impaired ${ }^{316}$. Further, different DC subsets may express different levels of cytokine receptors and, therefore, respond differently to stimulation by the family of alarmin cytokines (for example, IL-25, IL-33 and thymic stromal lymphopoietin (TSLP)) towards a type 2-promoting phenotype. Raising IL-25 or IL-33 levels in normally susceptible mice promotes resistance to T. muris infection ${ }^{317}$, and blocking TSLP signalling in normally resistant mice delays worm expulsion ${ }^{318}$. Other evidence implicating DCs as key players in the development of type 2 immunity comes from circadian studies, in which the DC circadian clock affected, at least in part, the outcome of T. muris infection ${ }^{319}$. Mice infected in the morning were more resistant to infection than mice infected at night. This time-of-day dependency in resistance to infection does not occur in transgenic mice in which DCs lack a core clock gene; the hypothesized mechanism is a circadian regulation of levels of type 1 immunity-promoting cytokines.
The potential immune regulatory effects of Trichuris spp. on inflammation in the large intestine ${ }^{65}$ have formed the basis of clinical trials using the pig whipworm T. suis (which causes an infection that generally does not persist beyond 6 weeks in the human intestine) to treat inflammatory diseases such as inflammatory bowel disease (IBD). To date, trials in which humans have orally ingested T. suis ova have shown no statistically significant benefits to patients with $\mathrm{IBD}^{75-77}$. Therapy with T. suis ova has also been evaluated in clinical trials in patients with several other inflammatory diseases, including rheumatoid arthritis, multiple sclerosis, psoriasis and food allergy, but none has shown clear clinical benefit ${ }^{78,79}$.

\section{Insights from animal models - type 2 immunity-controlled} effector mechanism. Mouse models also provide data on how $\mathrm{T}_{\mathrm{H}} 2$ cells stimulate worm expulsion (FIG. 4). Arguably, the effector mechanism supported by the largest amount of evidence is the role of goblet cells and mucus. Studies in mucin-deficient mouse strains ${ }^{80,81}$ have shown that mucin 2 and mucin 5 , subtypes $A$ and $\mathrm{C}$ are important in resistance to T. muris, probably via direct interactions with the parasite in the gut. The presence of mucin 2-degrading enzymes in the Trichuris spp. genome also supports an anthelminthic role for mucin ${ }^{82}$. Complementing a mucus-based effector mechanism, type 2 immunity cytokines can stimulate intestinal muscle contraction in the context of T. muris infection, and this increased contractility is associated with an acceleration of worm clearance ${ }^{83}$. Whereas increases in mucus production and contraction of gut muscles may be common host responses to most gastrointestinal helminths, regulation of epithelial cell turnover may be an effector mechanism specific to Trichuris spp. For example, the type 2 immunity cytokine IL-13 can increase the rate of epithelial turnover, thereby displacing the parasite from its intracellular niche ${ }^{84}$. Whether these effector mechanisms also apply to human trichuriasis is difficult to establish, although it is probable. Murine and human gastrointestinal helminth infections drive strong $\operatorname{IgE}$ responses, mostly non-specific ${ }^{85}$. As mentioned above, the levels of IgE antibodies specific to T. trichiura negatively correlate with worm burden in humans. Thus, individuals with light infection intensities have significantly higher anti-T. trichiura-specific IgE levels than those observed in individuals with heavy infection intensities $^{61}$. A direct role for IgE in host protection has been difficult to establish, and, instead of having a functional role, parasite-specific IgE levels in humans may represent a useful biomarker of a type 2 immune response. Animal models have revealed that B cells are important, although not essential, in resistance to T. muris infection $^{73,86}$. However, how B cells contribute to the protective immune response is unclear, and their contribution may not be related to their role in antibody production. Thus, B cells can also act as antigen-presenting cells ${ }^{87}$ and cytokine-producing regulatory cells ${ }^{88,89}$ and, therefore, could influence the development of either type 1 or type 2 immune responses and worm expulsion.

In humans with chronic trichuriasis and in mice infected with low numbers of eggs, regulation of the gut 
a

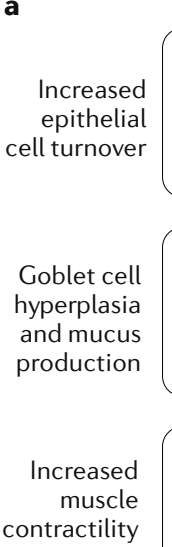

Colon

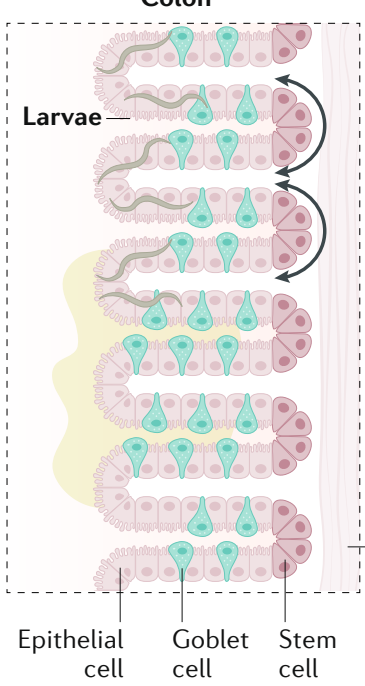

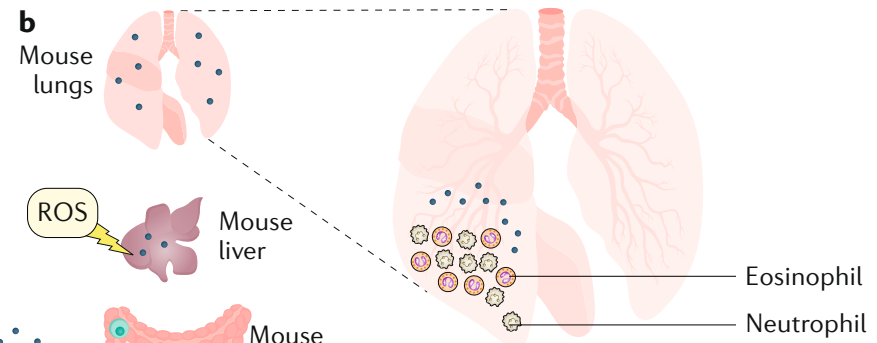

$\therefore \quad$ Mouse gut

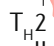
cell Muscle fibres
Intestinal immunity

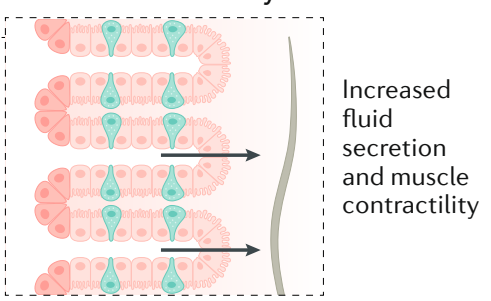

Fig. 4 | The anti-parasite effector mechanisms induced by the protective immune response. It is not known whether effector mechanisms similar to those observed in animal models also operate in humans, although it is a plausible hypothesis. a | In strains of mice resistant to Trichuris muris infection, the type 2 immunity cytokine IL-13 increases the rate of epithelial turnover, thereby displacing the parasite from its intracellular niche ${ }^{84}$. Resistance to infection also correlates with an increase in goblet cell numbers ${ }^{81}$. Through the use of mucin-deficient mouse strains, mucin 2 and mucin 5, subtypes $A$ and $C^{80,81}$ have been shown to be important in resistance to T. muris, probably via direct interactions with the parasite in the gut. Changes to gut physiology, including increased muscle contractility, are also thought to contribute to parasite expulsion. b | In mice resistant to Ascaris spp. infection, elimination of parasites from the gut involves the 'weep and sweep' mechanism (increased fluid secretion and muscle contractility) ${ }^{135}$. The mechanisms of lung immunity are unclear but probably involve type 2 immunity-controlled effector mechanisms. Both neutrophils and eosinophils infiltrate the lungs. Even less understood is liver immunity, although reactive oxygen species (ROS) have been implicated in the mechanism of resistance. $\mathrm{T}_{H} 2$ cell, type $2 \mathrm{~T}$ helper cell.

pathology induced by a large burrowing parasitic nematode is crucial in the maintenance of gut barrier function and prevention of sepsis. Regulation of pathology has been examined in some detail in the mouse model, and considerable evidence supports IL-10 as the regulatory cytokine vital in regulating IFN $\gamma$-mediated intestinal pathology and host protection ${ }^{90,91}$. Interestingly, in human trichuriasis, the quantitative trait locus on chromosome 9, mentioned above, contains genes that can influence IL-10 levels ${ }^{30}$. The cellular source of IL-10 is still debated, with FOXP3 $3^{+} \mathrm{T}_{\text {reg }}$ cells and other $\mathrm{CD} 4^{+}$ $\mathrm{T}$ cell populations as probable contributors ${ }^{68}$.

Insights from animal models - Trichuris spp. and their relationship with the microbiota. The close relationship between whipworms and the microbiota in the intestinal niche extends beyond the trigger for egg hatching ${ }^{54}$. The presence of Trichuris spp. infection alters both the numbers and the composition of the microbiota, and this alteration has been reported for T. muris in mice ${ }^{92,93}$ and T. suis in pigs ${ }^{94,95}$ and in some, but not all, human studies $^{96,97}$. Studies of T. muris in mice have revealed that for fitness the parasite has to acquire its own distinct microbiota from the host. The microbiota of T. muris is dominated by the Bacteroidetes and Firmicutes phyla, with a statistically significant rise in the proportion of bacteria of the Proteobacteria phylum that is not observed in the infected host microbiota ${ }^{98}$. Further, successful infections require the presence of host microbiota, and, remarkably, the T. muris-induced changes in the host microbiota may limit the success of subsequent infections ${ }^{98}$. In case of subsequent infections, parasite numbers are lower than the numbers from first-time infections, thereby providing a mechanism to limit host pathology and support chronicity of infection.

\section{Ascaris spp.}

Ascaris spp. eggs are very robust owing to their outer corticated coat (a layer of mammillated (with round protuberances) albuminous material) and can survive in the environment for long periods of time (estimates include up to 6 years in Germany and 14 years in Russia), although the majority of eggs probably die on shedding ${ }^{99}$. Evidence exists that tropical soils may be depleted of Ascaris spp. eggs and those of other STHs, including Trichuris spp., within 2 months, if no further contamination occurs ${ }^{100}$.

The life cycle of Ascaris spp. and the timing of each step have proved difficult to precisely define (FIG. 3). An early and extensive study in pigs ${ }^{101}$ described how, after hatching, larvae are released in the small intestine while still in the sheath of the first moult, and such L2 larvae migrate to the caecum and proximal colon, where they penetrate the mucosa. However, a more-recent study ${ }^{102}$ found that both the first and second ecdysis (moult) occur in the egg, before eggs hatch in the large intestine, and such retention of two moult sheaths is thought to be a feature favourable to parasite development. The L3 larvae then undergo a hepato-tracheal migration, a phenomenon that distinguishes the life cycle of Ascaris spp. 
from that of Trichuris spp. L3 larvae migrate via the portal blood vessels to the liver, where the L2 cuticle is shed and some larval growth occurs. Subsequently, L3 larvae leave the liver and advance to the lungs via the bloodstream, reaching first the heart and then the pulmonary vasculature ${ }^{99}$. In the lungs, the larvae penetrate the alveolar spaces and then migrate up the airway tree to the pharynx, where they are coughed up and swallowed. On their return to the small intestine, L3 moult to the fourth larval stage (L4 larvae) then undergo a final moult (L5) and develop to adult and sexually mature male and female worms ${ }^{103}$. Male and female adult worms measure $15-25 \mathrm{~cm}$ and $20-35 \mathrm{~cm}$, respectively, and their life expectancy has been estimated at $1-2$ years ${ }^{104}$. Adult worms produce unembryonated eggs that are shed in the faeces, where they develop to infectivity under appropriate conditions of temperature and moisture. The speed of the embryonation varies considerably according to the environmental conditions. For example, at $30^{\circ} \mathrm{C}$ embryonation takes $\sim 10-14$ days, whereas at $17^{\circ} \mathrm{C}$ it can take $45-55$ days $^{105}$. As it is the case with Trichuris spp. eggs, Ascaris spp. eggs that fail to embryonate are non-infective.

The reason why the life cycle of Ascaris spp. includes a difficult and risky hepato-tracheal migration is unclear, although some authors have argued that this migration confers fitness benefits to the parasite including increased size of adult worms ${ }^{106}$. What is clear is that larval migration contributes to both liver and lung pathol$\mathrm{ogy}^{107,108}$. Furthermore, the role of the liver in resistance to ascariasis is important but remarkably under-studied.

Human ascariasis - pathophysiology and immunology. Ascariasis is an excellent example of an infection that contributes to chronic morbidity; it particularly affects child growth via anorexia, malabsorption of nutrients and jejunal mucosal abnormalities, and it also has effects on cognitive development. The mechanisms underlying cognitive defects are not well understood but are probably nutritionally mediated, although the effect of systemic low-grade inflammation should not be disregarded. Owing to its large size, A. lumbricoides can also cause acute manifestations, including intestinal and biliary tract obstruction with related complications.

The relationship between humoral immune responses and $A$. lumbricoides infection in humans has been explored in various contexts ${ }^{109,110}$. Several studies have established a clear negative association between parasite-specific IgE levels and infection intensity. For example, a study of Nigerian children showed evidence of a statistically significant relationship between raised levels of parasite-specific IgE against the A. lumbricoides protein antigen ABA-1 and putative immunity in children ${ }^{111}$. Children with high IgE titres were less predisposed to heavy infection intensity than children with low titres, consistent with the association between elevated levels of parasite-specific IgE and reduced worm burdens observed in adults with trichuriasis. Furthermore, increased levels of inflammatory markers, such as C-reactive protein, were also detected in the putatively immune children compared with the infected children ${ }^{111}$. By contrast, another study found no relationship between humoral immune responses and current or future worm burdens ${ }^{112}$. The reason for these conflicting results is not clear. A. lumbricoides infection was also associated with a highly polarized $\mathrm{T}_{\mathrm{H}} 2$ cell-mediated response, with IL-4 and IL-5 responses predominating ${ }^{113}$. Two studies in Cameroonian children and adults provided further evidence of the role of cytokines produced by $\mathrm{T}_{\mathrm{H}} 2$ cells during ascariasis, including IL-5, IL-9, IL-10 and IL-13 $\left(\mathrm{REFS}^{63,114}\right)$. In contrast to the earlier of the two studies ${ }^{63}$, in the later study, the effects of cytokine production were more pronounced in children ${ }^{114}$. This finding led the authors to suggest that heterogeneity in cytokine responses may differ depending upon geographical location, owing to differences in transmission patterns or even historical differences in parasite dynamics. The authors concluded that these age-related and location-related differences may have implications for the differential effect of deworming programmes on immune responses. Finally, a study showed increased cytokine production by $\mathrm{T}_{\mathrm{H}} 2$ cells in children who had been repeatedly treated for A. lumbricoides infection, providing evidence that long-term treatment may increase $\mathrm{T}_{\mathrm{H}} 2$ cell-mediated anti-parasite immunity ${ }^{115}$.

Insights from animal models - the mouse model. Our understanding of the immunology of ascariasis is much more modest than that of the immunology of trichuriasis. One reason is the fact that there is no rodent model of ascariasis that demonstrates the entire life cycle of Ascaris spp. ${ }^{116}$. However, mouse models do provide insights into the factors that influence early stages of infection and larval migration ${ }^{116}$.

The mouse model enables an assessment of pathophysiological alterations under different parasitic burdens $^{117,118}$, genetic backgrounds ${ }^{118-121}$, host ages ${ }^{122}$ and egg infectivities ${ }^{122}$, and under repeated parasite exposure $^{44}$. The acute, early stages of infection are well established ${ }^{116,122}$ and demonstrate the physiological changes elicited by larval migration in the host, especially in the liver and lung. During larval migration in the liver, an intense inflammatory response is observed, particularly in resistant strains of mice ${ }^{120}$ (FIG. 4). Of note, proteomic analysis of hepatic tissues from resistant (CBA/Ca) and susceptible (C57BL/6J) mice strains infected with A. suum demonstrated intrinsic differences between the two strains, suggesting that resistance might be associated with the oxidative phosphorylation pathway and reactive oxygen species production ${ }^{121}$ and differential expression of components of the complement system ${ }^{118}$.

In primary infections with Ascaris spp., larval migration to the lungs promotes a local type 2 inflammatory response, marked by early production of IL- 5 followed by increased levels of IL-4, IL-5, IL-6, IL-33, CCL11 (also known as eotaxin), CCL2 (also known as MCP1) and CXCL10 (also known as IP10) and eosinophilia (excessive numbers of eosinophils in the blood) ${ }^{122-124}$. Interestingly, this elevated type 2 immune response was associated with a marked increase in IL-13 production by type 2 innate lymphoid cells and other type 2 immune cells, and it provided protection against the rodent hookworm Nippostrongylus brasiliensis ${ }^{125}$. 
This robust type 2 inflammatory response is associated with lung pathology, characterized by persistent airway hyper-responsiveness resembling an extreme form of allergic airway disease ${ }^{123}$. The severe impairment in respiratory function is aggravated by multiple exposures to the parasite despite the statistically significant reduction of parasitic burden ${ }^{44}$, which results in a reduction in larval migration to the liver and lungs. The inflammatory influx of cells in both the lung parenchyma and the bronchoalveolar fluid is initially dominated by neutrophils and correlates with IL-6 production in lung tissue $^{44,122,124}$. As the infection progresses, mononuclear cells accumulate at the inflammatory site in response to larval migration, produce $\mathrm{TNF}^{44,122}$ and ultimately differentiate into M2 macrophages in the type $2 \mathrm{immu}-$ nity environment ${ }^{124}$. Interestingly, parasite antigens can modulate macrophage differentiation, dendritic cell maturation ${ }^{126-128}$ (with further evidence of the ability of the parasite to modulate the immune response observed in experimental models of lipopolysaccharide-induced inflammation ${ }^{129}$ and autoimmune hepatitis ${ }^{130}$ ) and the immune response to heterologous (that is, other than from the parasite) antigens ${ }^{131}$ and viral co-infection ${ }^{132}$.

The protective inflammatory response observed in the mouse model of ascariasis may not be parasite-specific, given that pre-sensitization with heterologous allergens (from house dust mite) protects from a subsequent A. suum infection ${ }^{124}$. Conversely, pre-sensitization with Ascaris spp. antigens accelerates the mite-specific IgE response upon mite antigen inhalation ${ }^{133}$. These findings indicate the possible cross-reactivity between Ascaris spp. and arthropod antigens.

Insights from animal models - the pig model. Another important animal model for ascariasis is the A. suum pig model. Pigs are costly to maintain, and inbred and knockout porcine strains are currently unavailable. Nevertheless, given the economic burden of Ascaris spp. infection on the food industry and the fact that pigs are natural hosts for A. suum infection, understanding the pathophysiology of ascariasis in the swine model, particularly in the gastrointestinal phase of infection, is highly relevant. Of note, the use of the pig model enabled an understanding of both parasite-host interactions during establishment of the infection and the mechanisms of intestinal worm expulsion ${ }^{134,135}$. The initial phase of $A$. suum infection in pigs is very similar to the parasite migration observed in humans and induces both liver and lung pathology $y^{136-138}$. As observed in Ascaris spp. infections in humans and mice, production of IL-5, IL-13 and eotaxin and an intense eosinophilia are observed ${ }^{135,139}$. Blood basophilia (excessive numbers of basophils) and intestinal mastocytosis (accumulation of mast cells) are also common ${ }^{139-141}$ and may contribute to type 2 immunity induced by infection. Although the mechanisms by which Ascaris spp. parasites are expelled from the gut are less well defined than those of Trichuris spp., evidence suggests that elimination from the gut involves the 'weep and sweep' mechanism, an increase in fluid secretion and muscle contractility ${ }^{135}$. Further, there is some evidence in pigs naturally exposed to A. suum infection that continual exposure to infective larvae emerging from the eggs may inhibit larval migration from the intestine ${ }^{142}$. In the pig model, profound changes in the gut microbiota occur during A. suum infection, especially in the proximity of the initial site of egg hatching ${ }^{143}$. Thus, Ascaris spp. infection leads to a remarkable reduction in the gut microbial diversity, a reduction that is not related to worm burden. Moreover, the infection affects the abundance of specific microbial genera, particularly in the proximal colon ${ }^{143}$. The relevance of microbial composition alterations due to Ascaris spp. infection remains unknown. Finally, pathophysiological changes similar to those described in humans, mice and pigs have also been observed in other animal models including calves ${ }^{144}$, guinea pigs ${ }^{145}$, rabbits $^{146}$, gerbils ${ }^{147}$ and non-human primates ${ }^{148-150}$.

\section{Diagnosis, screening and prevention Clinical presentation}

Trichuriasis. Clinical disease is caused largely by inflammation of the caecum and large intestine, due to the presence of adult worms inducing a local inflammatory response and blood loss (from bleeding and oozing at the mucosal entry sites of worms) as they forage across the mucosa (FIG. 5a). Blood loss in trichuriasis has been estimated to be $0.005 \mathrm{ml}$ per worm per day ${ }^{151}$. Risk of anaemia is substantial among those with heavy infection intensities (defined as $\geq 800$ worms ${ }^{151}$ or $>5,000$ eggs per gram of stool (EPG $)^{152}$ ) or those co-infected with hookworm ${ }^{153,154}$. Clinical disease in individuals with T. trichiura infection is related to parasite burden. Most inhabitants (both children and adults) of endemic areas are infected with relatively few worms (that is, $<15$ adult worms ${ }^{155}$ ), and such infection intensities are associated with mild symptoms. Eosinophilia, if present, tends to be mild. However, in these regions, individuals are at risk of infection by other enteric parasites and are exposed to a range of environmental hazards. Non-specific symptoms of urticaria (itchy skin rashes), anorexia, abdominal pain and other gastrointestinal symptoms are difficult to attribute to any single cause, although they have been associated with $T$. trichiura infection ${ }^{156}$. By contrast, heavy infection intensities with several hundreds or even thousands of worms $s^{157,158}$ are often associated with colitis and substantial illness that may present as chronic iron deficiency anaemia in adults ${ }^{157}$, whereas children may present with failure to thrive, diarrhoea, which may be bloody, and short stature for their age, with or without symptoms of colitis or a severe illness. Even mild trichuriasis may be accompanied by growth retardation in children ${ }^{18}$, whereas TDS may be associated with severe malnutrition and growth stunting ${ }^{18,159}$. TDS, also known as massive infantile trichuriasis, is a severe illness associated with iron deficiency anaemia, chronic mucoid diarrhoea, rectal bleeding, rectal prolapse (a consequence of increased straining and/or peristalsis) and finger clubbing ${ }^{155,160}$. The pathogenesis of clubbing, a non-specific manifestation of many chronic diseases, is unknown but may relate to increased platelet-derived growth factor in the nail beds ${ }^{161}$. The triad of finger clubbing, rectal prolapse and chronic diarrhoea in children used to be pathognomonic of trichuriasis in endemic areas: $3-5 \%$ of children of 6 months to 6 years of age 

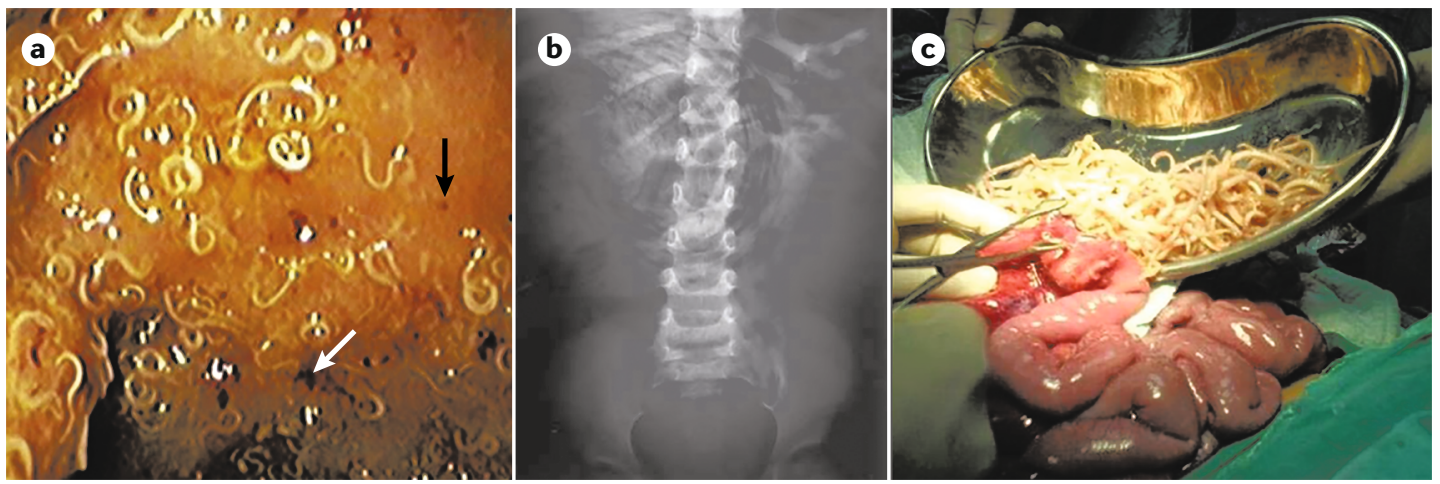

Fig. 5 | Clinical complications of trichuriasis and ascariasis. a | Colonoscopic image from a 15-year-old girl with Trichuris dysentery syndrome (TDS). The colonic mucosa is covered with parasites (seen are posterior ends tethered to the mucosa). Note petechial (black arrow) lesions and blotchy mucosal haemorrhages (white arrow). b | Abdominal plain radiograph demonstrating 'tramline' appearance caused by a heavy intestinal infestation by Ascaris lumbricoides. The duodenum is packed with worms, resulting in a whorled (spiral) appearance of black radiolucent worms outlined by gas. $\mathbf{c} \mid$ Small-bowel obstruction by A. lumbricoides. The image shows a mass of A. lumbricoides worms collected from a section of obstructed small intestine following enterotomy in a 3-year-old boy in South Africa. Part a is adapted with permission from REF. ${ }^{157}$, Elsevier. Part $\mathbf{b}$ is adapted from REF. ${ }^{311}$, Springer Nature Limited. Part $\mathbf{c}$ is adapted from REF. ${ }^{312}$, CC BY 3.0 (https://creativecommons.org/licenses/by/3.0/).

were estimated to have recurrent rectal prolapse in a region of the Carribean ${ }^{162}$. However, with improvements in environmental hygiene and access to anthelminthics, TDS and rectal prolapse are now infrequent. TDS has more recently been recognized as a problem in adults presenting with severe iron deficiency anaemia ${ }^{157}$, and this observation probably reflects poor clinical recognition of trichuriasis in adults living in conditions of severe poverty and who are not included in anthelminthic treatment programmes. Heavy infection intensities may be associated with increased intestinal permeability and a chronic inflammatory response, as indicated by elevated circulating levels of the pro-inflammatory cytokine $\mathrm{TNF}^{163}$.

T. trichiura may be a chance finding in individuals undergoing colonoscopy for abdominal pain and altered bowel habits ${ }^{164,165}$. In individuals with heavy infection intensities, colonoscopy shows numerous motile worms tethered to the intestinal mucosa by their anterior ends ${ }^{157,165}$. Histopathology of the large intestine in patients with trichuriasis often shows only mild changes, with increased numbers of inflammatory cells in the lamina propria, particularly in adults ${ }^{157,159}$, whereas in children histological changes range from mild inflammation to localized cryptitis at worm attachment sites to a highly inflamed intestinal mucosa that is oedematous, eroded and friable ${ }^{64,158}$. In individuals with heavy infection intensities, adult worms may be found from the caecum to the rectum, and the mucosa is studded with bleeding points representing previous mucosal entry points of foraging adult worms ${ }^{157,159}$.

Prolonged mucosal bleeding and inflammation affect the nutritional status of children, particularly those on marginal diets (that is, low in iron and other essential nutrients $)^{152}$. Further, the presence of adult worms may also affect nutrient absorption through mucosal damage or disruption of the intestinal microbiota, although evidence for an effect of disrupted microbiota is limited ${ }^{97,166}$. Damaged mucosa may have increased susceptibility to infections with other intestinal pathogens with which $T$. trichiura has been associated, such as Entamoeba histolytica ${ }^{167}$. T. trichiura infection has been shown to correlate with the presence of both A. lumbricoides and Campylobacter spp. ${ }^{97,168}$. Whether multiple intestinal infections are simply coincidental or whether they influence each other's pathogenicity in humans is unclear $^{169}$, although exacerbated disease and pathology have been reported in pigs co-infected with T. suis and Campylobacter jejuni ${ }^{170}$.

Ascariasis. In endemic areas, the majority of A. lumbricoides infections cause mild or no symptoms. Clinical disease is restricted to a small percentage of individuals with a high parasite burden, as most individuals harbour only a few worms ${ }^{171,172}$, although there are no upto-date figures on the percentage of clinical cases. The clinical manifestations are directly related to the parasite life cycle and depend on the infection intensity. During larval migration through the airways (10-14 days after infection), classic respiratory alterations, including lung infiltration (visible on the chest radiograph), intense eosinophilia, cough and wheeze are observed and are known as Löffler syndromes ${ }^{173}$. Urticaria, cough, dyspnoea, haemoptysis (coughing up blood) and abnormal breath sounds on auscultation are also non-pathognomonic signs associated with larval migration through pulmonary tissue. Adult parasites dwelling in the small intestine can induce, depending on their numbers, various gastrointestinal outcomes, including upper gastrointestinal bleeding, small-bowel obstruction (FIG. 5b,c), volvulus (twisting of an intestinal tract that results in obstruction and/or bowel ischaemia), intussusception (folding of the intestinal tract into the section of intestine that is immediately ahead), peritonitis, haemorrhagic infarction of the bowel and perforation ${ }^{174,175}$. Adult worms may also migrate to extra-intestinal sites, and hepatobiliary and pancreatic ascariasis (HPA) may then occur, leading to biliary colic, acute cholecystitis, acute 
pancreatitis, acute cholangitis (inflammation and/or infection of the biliary tree) and hepatic abscess ${ }^{176}$. Intestinal perforation and peritonitis ${ }^{177}$, often following appendicular ascariasis ${ }^{178}$, is a rare but severe and often fatal surgical emergency caused by ascariasis in endemic areas. Heavy infection intensities with ascariasis are a common cause of surgical emergencies in endemic regions, and these emergencies are primarily caused by obstruction of the narrow intestinal lumen of a young child with a bolus of $A$. lumbricoides worms ${ }^{179}$.

Asthenia (physical weakness), lack of appetite, abdominal pain and/or distension, nausea, diarrhoea and weight loss are common in children with severe intestinal ascariasis in endemic areas ${ }^{176}$. Moderate to heavy infection intensities in children have been associated with impairment in physical and mental development ${ }^{180}$ and may also contribute to malnutrition ${ }^{181}$ and deficiency of vitamins $A$ and $C^{182}$.

\section{Diagnosis of trichuriasis and ascariasis}

As with other STH infections, the laboratory diagnosis of ascariasis and trichuriasis relies on the examination of a stool sample to determine the presence and, whenever possible, the quantity of parasite eggs. Currently, the WHO recommends the use of the Kato-Katz method to examine stool samples for STH infections by direct microscopy ${ }^{183}$ and to assess two slides per sample ${ }^{184}$. Other parasitological methods include formol-ether concentration, McMaster, FLOTAC and Mini-FLOTAC, and the sensitivity of all these methods varies according to the infection intensity ${ }^{185}$ (TABLE 1). Direct observation of saline smears, although of low sensitivity, is the most widely used microscopic method to examine stools. New parasitological methods, such as mobile phone microscopy ${ }^{186}$ and FECPAKG2 (a sedimentation method coupled with imaging and remote identification and quantification of STHs) ${ }^{187}$ have been developed but require extensive evaluation.

Considering the reduced sensitivity of microscopybased parasitological methods, molecular-based diagnostic assays have been developed, aiming to improve sensitivity and specificity. The reported sensitivities of molecular methods are superior to those of microscopy-based methods for the diagnosis of both ascariasis ${ }^{188-191}$ and trichuriasis ${ }^{190,191}$, despite the lack of an adequate gold standard for comparison ${ }^{192}$. Of note, most molecular-based assays have been developed as multiplexed ${ }^{193-195}$ or multi-parallel assays ${ }^{189,196,197}$ for detection of different parasites. However, the development of these methods is hampered by the requirement for specific, more-expensive equipment and the lengthy procedure of DNA extraction from the stool samples, both of which may limit the application of molecular diagnostic assays in field settings. A colorimetric isothermal assay, consisting of a one-step DNA amplification method, has also been developed for the diagnosis of ascariasis and trichuriasis, combining high sensitivity and high tolerance to substances present in faecal samples that might interfere with the assay ${ }^{198}$ (such as complex polysaccharides, salts, lipids and urate, among others ${ }^{199}$ ), and might be a promising tool for diagnosis in the field. Nevertheless, at present, molecular-based assays remain restricted to research groups working in specialized laboratories, and none of them has been rolled out for routine use in endemic regions. Thus, for the moment, microscopy-based analysis remains the mainstay for the diagnosis of ascariasis and trichuriasis - there is still considerable demand for such diagnostics from physicians in local hospitals and clinics and the mothers of children living in communities in endemic areas, who often attribute a large amount of illness in their children to the presence of these parasites.

Advantages and limitations of diagnostic methods. Microscopy-based and molecular-based diagnostic methods are only effective after infections have become patent. Microscopy-based methods have very limited sensitivity for infections of light intensity ${ }^{185}$, with the intensity of T. trichiura and A. lumbricoides infections estimated and classified as light (1-4,999 EPG and 1-1,000 EPG, respectively), moderate (5,000-49,999 EPG and 1,001-9,999 EPG, respectively) or heavy ( $\geq 50,000$ EPG and $\geq 10,000$ EPG, respectively), according to the WHO classification ${ }^{200}$. By contrast, molecularbased assays, although more-expensive and of limited field applicability, enable detection of infections of low intensity in areas where anthelminthic control programmes have reduced prevalence and infection intensity to very low levels and where local or regional elimination strategies are being considered. The use of more-sensitive assays, such as quantitative PCR, at central laboratories might be justified under such circumstances, despite the need for sophisticated and costly equipment and trained personnel. Low-cost, field-applicable assays (such as lateral flow assays to detect specific parasite antigens in stool samples) are presently not available but would increase considerably the effectiveness of control programmes, by assisting in making decisions about the frequency of anthelminthic treatment and what population groups should receive treatment.

\section{Prevention of trichuriasis and ascariasis}

The prevention of trichuriasis and ascariasis, as well as any other STH infections, relies on the combination of several approaches that reduce prevalence. Among them, the WHO guidelines on preventive chemotherapy based on MDA in endemic areas aim to reduce the morbidity in preschool and school-age children by lowering the prevalence of infections of moderate and heavy intensity ${ }^{11}$. Preventive chemotherapy is an important tool for reduction of prevalence and morbidity of both diseases, with a reduction of up to $80 \%$ in the overall parasite burden and prevalence in endemic areas ${ }^{201-203}$. In 2017, a WHO Guideline Review Committee revisited the previous guidelines and provided updated global, evidence-informed recommendations on preventive chemotherapy ${ }^{8}$ in areas endemic for STHs; however, this approach represents a short-term strategy for control of helminth infections, as reinfection often occurs in endemic areas in the absence of clean water, sanitation and hygiene ${ }^{204}$. The water, sanitation and hygiene (WASH) programme advocates improvements in access to water (in terms of water quality and 
Table 1 | Diagnostic methods for Trichuris trichiura and Ascaris lumbricoides infections

\begin{tabular}{|c|c|c|c|c|c|c|c|}
\hline \multirow[t]{2}{*}{ Test } & \multirow[t]{2}{*}{ Output } & \multicolumn{2}{|c|}{ Sensitivity (\%) } & \multicolumn{2}{|c|}{ Specificity (\%) } & \multirow[t]{2}{*}{ Advantages } & \multirow[t]{2}{*}{ Limitations } \\
\hline & & T. trichiura & A. lumbricoides & T. trichiura & A. lumbricoides & & \\
\hline $\begin{array}{l}\text { Direct } \\
\text { microscopy }\end{array}$ & Egg detection & 62.8 & 52.1 & 97.5 & 99.6 & Low cost & $\begin{array}{l}\text { Positive results only at } \\
\text { high parasitic burden }\end{array}$ \\
\hline Kato-Katz ${ }^{185,303-305}$ & $\begin{array}{l}\text { Egg detection } \\
\text { and egg } \\
\text { quantification }\end{array}$ & $62.8-91.0$ & $56.9-88.1$ & 97.5 & 99.6 & $\begin{array}{l}\text { Relatively low } \\
\text { cost; possible to } \\
\text { determine the } \\
\text { burden of infection }\end{array}$ & $\begin{array}{l}\text { Overall low sensitivity } \\
\text { (especially at light } \\
\text { infection intensities); } \\
\text { need for a qualified } \\
\text { microscopist }\end{array}$ \\
\hline Formol-ether ${ }^{185}$ & Egg detection & 81.2 & 56.9 & 97.5 & 99.6 & Relatively low cost & $\begin{array}{l}\text { Does not detect } \\
\text { unembryonated eggs of } \\
\text { Ascaris spp. }\end{array}$ \\
\hline FLOTAC $^{305}$ & $\begin{array}{l}\text { Egg } \\
\text { quantification }\end{array}$ & $88.7-100$ & 79.7-100 & 97.5 & 99.6 & $\begin{array}{l}\text { Detection of } \\
\text { different STHs } \\
\text { simultaneously }\end{array}$ & $\begin{array}{l}\text { Requires centrifugation } \\
\text { steps with two different } \\
\text { rotors; relatively high cost }\end{array}$ \\
\hline Mini-FLOTAC ${ }^{306}$ & $\begin{array}{l}\text { Egg } \\
\text { quantification }\end{array}$ & $76.2-91.5$ & $63.3-75.5$ & 97.5 & 99.6 & $\begin{array}{l}\text { Detection of } \\
\text { different STHs } \\
\text { simultaneously }\end{array}$ & $\begin{array}{l}\text { Limited sensitivity } \\
\text { (sensitivity comparable } \\
\text { to that of the Kato-Katz } \\
\text { method but lower than } \\
\text { that of FLOTAC) }\end{array}$ \\
\hline FECPAK $^{306}$ & $\begin{array}{l}\text { Egg } \\
\text { quantification }\end{array}$ & $59.8-65.8$ & 58.9-75.6 & 97.5 & 99.6 & $\begin{array}{l}\text { Detection of } \\
\text { different STHs } \\
\text { simultaneously; } \\
\text { simple procedure } \\
\text { with results within } \\
\text { an hour }\end{array}$ & $\begin{array}{l}\text { Requires internet } \\
\text { connection }\end{array}$ \\
\hline McMaster $^{303,307}$ & $\begin{array}{l}\text { Egg } \\
\text { quantification }\end{array}$ & $61.1-81.8$ & $75.6-80.3$ & 97.5 & 99.6 & $\begin{array}{l}\text { Relative low cost; } \\
\text { enables quick } \\
\text { counting of eggs; } \\
\text { may be applicable } \\
\text { for monitoring } \\
\text { large-scale } \\
\text { treatment } \\
\text { programmes }\end{array}$ & $\begin{array}{l}\text { Need for a special } \\
\text { counting chamber }\end{array}$ \\
\hline $\begin{array}{l}\text { LAMP } \\
\text { assay }^{192,198,308}\end{array}$ & $\begin{array}{l}\text { Identification } \\
\text { of DNA from } \\
\text { Ascaris spp. or } \\
\text { Trichuris spp. }\end{array}$ & 100 & $96.3-100$ & 100 & 100 & $\begin{array}{l}\text { Possible to detect } \\
\text { multiple infections } \\
\text { by multiplexed } \\
\text { assays; high }\end{array}$ & $\begin{array}{l}\text { Risk of low sensitivity } \\
\text { due to the presence of } \\
\text { inhibitors in the faecal } \\
\text { sample; decreased }\end{array}$ \\
\hline qPCR ${ }^{306}$ & $\begin{array}{l}\text { Quantification } \\
\text { of DNA from } \\
\text { Ascaris spp. or } \\
\text { Trichuris spp. }\end{array}$ & $72.3-100$ & $85-100$ & 100 & 100 & specificity & $\begin{array}{l}\text { sensitivity it formalin } \\
\text { is used for fixation } \\
\text { of samples; requires } \\
\text { specialized equipment } \\
\text { and has restricted use } \\
\text { in the field }\end{array}$ \\
\hline
\end{tabular}

A. lumbricoides, Ascaris lumbricoides; LAMP, loop-mediated isothermal amplification; qPCR, quantitative PCR; STHs, soil-transmitted helminths; T. trichiura, Trichuris trichiura.

quantity and distance to water) and sanitation (in terms of access to latrines, their proper maintenance and faecal waste management), the use of hygiene practices and changes in behaviour related to environmental and personal hygiene ${ }^{205,206}$. Reduced odds of $A$. lumbricoides and T. trichiura infections are associated with treated water, access to sanitation and hygiene procedures (such as hand washing before eating and after defaecation and the use of soap) ${ }^{207}$. However, there is an urgent need to gather stronger evidence to support the role of WASH programmes in the control of STH infections ${ }^{208}$.

Preventive chemotherapy. In contrast to most regimens for individual patient management, preventive chemotherapy programmes, advocated since 2001 by the WHO, rely on single-dose treatment (TABLE 2). Preventive chemotherapy involves periodic administration of recommended anthelminthics, usually a single dose of oral albendazole or mebendazole to preschool and school-age children, women of reproductive age (as well as pregnant women in the second and third trimesters and lactating mothers) and adult groups with high risk of exposure to STH infections (for example, tea pickers). The recommended treatment schedule (once or twice per year) is determined by the initial prevalence of STHs ${ }^{11,209}$. The goal of preventive chemotherapy programmes was to achieve a minimum coverage of $75 \%$ of the most affected groups by 2020. In 2017, >598 million children were treated in endemic regions, corresponding to $69 \%$ of all children at risk ${ }^{208}$, indicating that the 2020 targets are nearly met. Thus, the WHO set new targets and indicators ${ }^{210}$ : to achieve and maintain elimination of STH morbidity (defined as the prevalence of moderate and heavy infection intensities below $2 \%$ ) in 
preschool and school-age children by 2030; to reduce the number of drug tablets needed in preventive chemotherapy for STH infections; to increase domestic financial support for preventive chemotherapy for STH infections; to establish an efficient STH infections control programme in adolescent, pregnant and lactating women; to establish an efficient strongyloidiasis (an STH infection caused by Strongyloides spp. nematodes) control programme in school-age children; and to ensure universal access to at least basic sanitation and hygiene by 2030 in STH-endemic areas.

Epidemiological surveillance. The high-throughput performance of serological assays indicates the suitability of these tools in epidemiological surveillance. The development of serological tools to improve the detection of prepatent infections could improve the effectiveness of surveillance during elimination programmes. However, the development of such assays is largely hampered by the lack of antigen specificity - owing to cross-reactivity between helminth antigens ${ }^{211-214}$, and even between helminth and arthropod (such as mosquito and tick) antigens $^{215,216}$ - and the inability to discriminate between past and current infections. Although serological assays are available for the diagnosis of animal infections ${ }^{217-220}$, serological assays of human infections are restricted to detection of $A$. suum ${ }^{221}$ in humans. Of note, anti-A. suum IgY formed immune complexes with $A$. lumbricoides antigens in serum from patients with ascariasis, with diagnostic sensitivity and specificity values of $80 \%$ and $90 \%$, repectively ${ }^{222}$, and, therefore, showed potential for immune-based diagnosis of ascariasis. Although on the one hand antigen cross-reactivity reduces the ability to discriminate among helminth infections, on the other hand serological assays using cross-reactive or conserved epitopes among different helminths would be useful for the control of STH infections, particularly in the application and assessment of parasite control achieved using MDA (see Outlook).

Vaccines. Vaccines might reduce the parasite burden and, consequently, the morbidity and transmission of infection (see Outlook). Evidence from experimental murine models indicated that continuous exposure to A. suum eggs (three successive infections with 2,500 eggs each) led to up to $98 \%$ protection, measured as larval reduction in the host tissues ${ }^{44,223}$. In mice, immunization with extract of adult T. muris worms or excretedsecreted proteins induced a high degree of protection (up to $100 \%$ larval reduction) ${ }^{224,225}$. The development of vaccines using defined antigens against $A$. lumbricoides and T. trichiura has been pursued, but it is still restricted to experimental models, and no vaccines against A. lumbricoides or T. trichiura are currently being assessed in clinical trials. The selection of new vaccine candidates and the understanding of protective mechanisms induced by immunization might open new perspectives for the control of these infections in endemic areas, as individual or combined ('pan-helminth') vaccines ${ }^{226}$.

\section{Management}

As well as their prevention, the control and treatment of ascariasis and trichuriasis, like other STH infections, can be achieved through various strategies that include environmental sanitation and hygiene, health education and the use of anthelminthic drugs. Environmental sanitation and hygiene are effective, but appreciable reductions in prevalence and infection intensity take time. Indeed, it is difficult to distinguish the effects of WASH initiatives from those of anthelminthic drugs in control programmes ${ }^{208}$. Improved methods to assess levels of environmental exposure to STHs and of uptake and usage of WASH directives will contribute to understanding the role of WASH programmes as adjuncts to deworming programmes. By contrast, the use of effective and safe anthelminthic drugs has been shown to be effective and rapid in reducing prevalence, intensity and morbidity of these infections. Treatment of ascariasis and trichuriasis includes management of diagnosed patients with the aim of cure and MDA of anthelminthic drugs to populations in endemic areas to reduce the burden of disease (preventive chemotherapy).

The current drugs recommended by the WHO for the treatment of STH infections are albendazole, mebendazole, levamisole and pyrantel pamoate ${ }^{11,227}$. Albendazole and mebendazole are the two benzimidazoles that have been used most widely for decades against STHs in the treatment of individual patients and in MDA programmes. For MDA programmes, millions of tablets are donated each year.

Table 2 | Recommended preventive chemotherapy regimens and efficacy of anthelminthic drugs

\begin{tabular}{|c|c|c|c|c|c|c|c|}
\hline \multirow[t]{2}{*}{ Treatment } & \multirow{2}{*}{$\begin{array}{l}\text { Mechanism of } \\
\text { action }\end{array}$} & \multicolumn{3}{|c|}{ T. trichiura infection } & \multicolumn{3}{|c|}{ A. lumbricoides infection } \\
\hline & & $\begin{array}{l}\text { Preventive } \\
\text { chemotherapy }^{a}\end{array}$ & $\begin{array}{l}\text { Cure rate } \\
(\%)^{\mathrm{b}}\end{array}$ & $\begin{array}{l}\text { Egg reduction } \\
\text { rate }(\%)^{b}\end{array}$ & $\begin{array}{l}\text { Preventive } \\
\text { chemotherapy }^{\mathrm{a}}\end{array}$ & $\begin{array}{l}\text { Cure rate } \\
(\%)^{\mathrm{b}}\end{array}$ & $\begin{array}{l}\text { Egg reduction } \\
\text { rate }(\%)^{b}\end{array}$ \\
\hline Albendazole & $\beta$-Tubulin binding & Once & 32.1 & 64.3 & Once & 96.5 & 99.7 \\
\hline Mebendazole & $\beta$-Tubulin binding & Once & 44.4 & 80.7 & Once & 96.8 & 99.5 \\
\hline $\begin{array}{l}\text { Albendazole- } \\
\text { ivermectin }\end{array}$ & NA & Once & 60.0 & 95.5 & Once & 96.7 & 99.9 \\
\hline Levamisole & $\begin{array}{l}\text { L-subtype } \mathrm{nAChR} \\
\text { agonist }\end{array}$ & Once & 23.4 & 41.8 & Once & 93.0 & 97.0 \\
\hline Pyrantel pamoate & $\begin{array}{l}\text { L-subtype nAChR } \\
\text { agonist }\end{array}$ & Once & 28.5 & 62.3 & Once & 97.5 & 91.7 \\
\hline
\end{tabular}

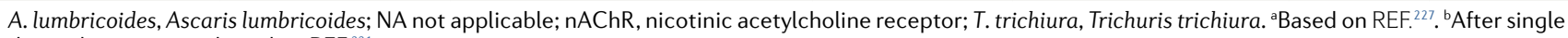
dose administration, based on REF. ${ }^{231}$ 


\section{Treatment of trichuriasis}

Although the recommended monotherapies are effective when used at single oral doses for preventive chemotherapy, none of them shows acceptable efficacy (egg reduction rates $>90 \%$ based on the target product profile, which describes the desired drug characteristics, for drugs used for STHs) ${ }^{228}$ against T. trichiura infections at single doses (TABLE 2). However, the efficacy increases when the drugs are used in the recommended dosing schedules. A double-blind clinical study on Pemba Island, Tanzania, showed that mebendazole treatment (twice a day for 3 days) achieved considerably higher cure and egg reduction rates against T. trichiura infections than single-dose treatment (cure rate $42.9 \%$ versus $6.8 \%$ and egg reduction rate $98.1 \%$ versus $71.7 \%$ ) in school-age children ${ }^{229}$. Why anthelminthic drugs are less effective against $T$. trichiura infections than against other STH infections is not known, but the location of the parasite (as discussed in the Outlook section) might have a role.

Treatments under investigation. Owing to this low efficacy of standard monotherapy, monodose combination chemotherapy has been widely advocated, which includes the advantages of a single administration and drug combination therapy. Since 2017, albendazoleivermectin is on the WHO essential medicine list for the treatment of STH infections including strongyloidi$\operatorname{asis}^{230}$. This drug combination was classified as high priority combination ${ }^{231}$, as this treatment is already widely used for lymphatic filariasis (an infection caused by the bite of a mosquito carrying nematodes of the Filarioidea family). Despite the large scale use of this drug combination, the available efficacy data for STH infections are limited ${ }^{232}$, and a multi-country randomized controlled double-blind trial has been launched to provide more robust evidence on the efficacy and safety of co-administration of ivermectin and albendazole ${ }^{233}$.

As moxidectin has been approved for the treatment of onchocerciasis (also known as river blindness, an infection caused by the bite of blackflies carrying the nematode Onchocerca volvulus) by the $\mathrm{FDA}^{234}$, albendazole-moxidectin might serve as an alternative drug combination to albendazole-ivermectin. Albendazolemoxidectin, used at the recommended dosages, was safe and effective against $T$. trichiura infections ${ }^{235}$. As for albendazole-ivermectin, large-scale trials to establish the effectiveness of albendazole-moxidectin are necessary ${ }^{235}$.

In contrast to the recommended treatments, ivermectin $^{236}$ or moxidectin ${ }^{235}$, oxantel pamoate is more effective, having excellent trichuricidal properties ${ }^{237}$. To compensate for lack of efficacy of oxantel against A. lumbricoides and hookworm, oxantel has been combined with pyrantel pamoate (for example, Quantrel (Johnson \& Johnson)). Several clinical trials have successfully demonstrated that a combination of albendazole and oxantel pamoate is safe and efficacious ${ }^{238}$. A network meta-analysis found that a single dose of this combination was associated with an estimated cure rate of $88.7 \%$ and an egg reduction rate of $96.7 \%^{231}$. Efforts are ongoing to determine whether any existing data on oxantel pamoate (from veterinary medicine, in which the drug is widely available, or from regions where it is registered for use in humans, for example, the Philippines) can be utilized to support registration of oxantel pamoate with the European Medicines Agency and/or the FDA, with the ultimate goal of using it as combination drug in treatment campaigns.

Emodepside, a veterinary anthelminthic licensed under the names Profender (Bayer) and Procox (Bayer), is the only drug in the late stages of clinical development, in the depleted drug development pipeline for STH infections. Emodepside is a cyclo-octadepsipeptide that targets the evolutionarily conserved calcium-activated potassium channel slowpoke 1 (SLO-1) and the latrophilin receptors LAT-1 and LAT-2 $\left(\mathrm{REF}^{239}\right)$, which are involved in nematode neuromuscular function. The drug is currently undergoing clinical testing against onchocerciasis. In laboratory models of STH infections, emodepside showed a broad spectrum of activity against the major STHs ${ }^{240}$. Thus, emodepside should also be considered for development as an anthelminthic drug, although it has the disadvantage of high production costs, as it is a semi-synthetic compound whose precursor is a metabolite of the fungus Mycelia sterilia. Testing of other SLO-1 inhibitors is currently ongoing.

Anthelminthic drug resistance. To date, there is no evidence of resistance to benzimidazoles in humans ${ }^{241}$. However, the drug selection pressure that led to widespread anthelminthic drug resistance in veterinary helminths is now similar for human STHs, owing to the large-scale use of preventive chemotherapy. The reasons why it is difficult to assess the presence of anthelminthic drug resistance in humans include the variable drug efficacy, the lack of validated phenotypic or genotypic tests to detect resistance and working with difficult sample matrices (that is, stool). Efforts are ongoing to develop molecular and genomic screening tests of human STHs to identify mutations that are likely to be associated with benzimidazole resistance, on the basis of our understanding of resistance in veterinary helminths. Several initiatives are ongoing, including the STOP, DeWorm3 and Starworms (stop anthelmintic resistant worms) projects, that focus on the assessment of drug efficacy and the development of molecular methods for the detection of anthelminthic drug resistance ${ }^{230}$. It is important to monitor the presence of resistance-associated singlenucleotide polymorphisms in human STHs before resistance becomes clinically established.

\section{Treatment of ascariasis}

Several anthelminthics have been developed to effectively manage ascariasis, although their long-term effectiveness remains a concern. Albendazole, mebendazole, levamisole and pyrantel pamoate have high efficacy against $A$. lumbricoides in terms of both cure rates and egg reduction rates even with a single dose ${ }^{242}$ (TABLE 2). Several other marketed anthelminthics, such as ivermectin (TABLE 2), moxidectin and tribendimidine, are also highly effective against $A$. lumbricoides ${ }^{242}$.

Clinical disease resulting from ascariasis in children and adults includes intestinal obstruction (a common occurrence in children in endemic areas), peritoneal 
ascariasis (due to the migration of $A$. lumbricoides larvae into the peritoneum through damaged intestine (such as an ulcer from typhoid fever, an inflamed appendix or, occasionally, an apparently healthy bowel)) and HPA, which commonly occurs in adults.

Conservative treatment is the mainstay in the management of patients with HPA and involves managing clinical signs and symptoms with appropriate treatment, such as bowel rest (that is, refraining from eating anything by mouth for a few days as required), intravenous fluids, analgesic antispasmodics and antibiotics followed by albendazole ${ }^{243}$. If adult worms are not expelled from the ductal lumen by 3 weeks after anthelminthic treatment, worms may be extracted by endoscopic retrograde cholangio-pancreatography (with endoscopic exploration of the bile and pancreatic ducts and extraction of worms), sphincterotomy (enlargement of the bile duct opening) or, occasionally, laparotomy ${ }^{244}$. Intestinal obstruction can often be managed conservatively, although those with signs of peritoneal inflammation generally require surgery to relieve the obstruction or to resect damaged bowel ${ }^{245}$.

\section{Quality of life}

\section{Estimating the burden of STH infections}

Cross-sectional and prospective observational studies from the 1990s or earlier indicated substantial adverse long-term effects of STH infections on several nutritional indices, such as stunting, and also on childhood cognitive development ${ }^{246}$. However, randomized controlled trials have been more equivocal in showing effects of STH infections on nutritional and cognitive indices, as these trials have shown conflicting results or results that did not reach statistical significance; more recent systematic reviews of intervention studies have been able to demonstrate only negligible effects on growth and nutritional parameters, cognition and mortality ${ }^{247-249}$. A meta-analysis of observational and randomized treatment studies showed no overall effect on cognitive parameters in children in treatment trials but infection-related deficits in some parameters in observational studies, although these deficits were considered to be highly vulnerable to bias because of the poor methodological quality of many studies ${ }^{250}$. A systematic review of the benefits of nutritional supplementation (for example, iron) in addition to anthelminthic treatment highlighted that the evidence base was so weak that no nutritional supplementation could be recommended ${ }^{251}$. Criticisms of these systematic reviews have focused largely on the following considerations: that including uninfected children or children with low parasite burdens in the analyses might have 'diluted' the effects of STH infections on the parameters measured; that the study populations may have been infected with a variety of different helminth species and, therefore, it would have been impossible to attribute species-specific effects; and that school absenteeism by the most affected children could have biased the results towards no effect, as these children would not have been examined as they did not attend school. A critical appraisal of systematic reviews noted the need for new studies designed and powered to overcome these limitations to enable morbidity from STH infections to be measured more accurately ${ }^{246}$. Certainly, observational studies in children with heavy infection intensities have shown remarkable effects of treatment on 'catch-up' growth (compensatory accelerated growth after a period of slow development) after treatment, particularly in those with severe triuchuriasis ${ }^{157,252,253}$, but the frequencies of children at risk of severe disease have declined markedly in line with worldwide reductions in poverty rates ${ }^{254,255}$.

\section{Trichuriasis}

In keeping with the challenges in quantifying the effects of living with STH infections outlined above, estimating the effects of trichuriasis on quality of life in populations in endemic areas is complicated by uncertainty in the estimates of prevalence and parasite burdens and imprecision in those effects on quality of life indices. Quality of life is most likely to be affected during chronic infections and/or infections of heavy intensity. Death is thought to be an unusual outcome, although no reliable estimates of mortality exist ${ }^{256}$. Nevertheless, trichuriasis probably has direct effects on several quality of life domains, such as economic productivity, educational performance and health, although there are limited data measuring such effects. Trichuriasis can affect $\operatorname{cognition}^{257}$, school performance ${ }^{258}$ and school absenteeism rates ${ }^{259}$ and, therefore, probably has direct effects on educational achievement and economic potential of individuals. Curative chemotherapy and treatment with iron in children with TDS can have dramatic effects on linear growth velocities ${ }^{155}$. The benefits of deworming programmes in children has generated considerable controversy given negative findings of meta-analyses ${ }^{248}$, as discussed above. However, T. trichiura infection may impair developmental and cognitive abilities in children, although the benefits of treatment in reversing such deficits is hotly debated ${ }^{158,248,250,260}$.

Health effects such as those associated with anaemia and poor growth will probably affect physical fitness $^{261}$ and economic productivity ${ }^{262}$, as well as the quality of social interactions and well-being. Anaemia can be severe in vulnerable groups (such as pregnant women, whose iron reserves may be severely depleted), although it is usually not as pronounced as in those with hookworm infection ${ }^{154,157}$. The various health consequences of infection can be summarized crudely in disability-adjusted life years (DALYs), an estimate of the number of years of 'healthy life' that are lost and are attributable to a specific infection, on the basis of morbidity and mortality data. For trichuriasis, estimated DALYs are highly variable between studies but were estimated at 0.213 million in 2017 (REF. ${ }^{263}$ ), with the greatest burden in the populous regions of Asia ( $60 \%$ of DALYs). This figure represents a decline of $23 \%$ since 2007, largely due to reductions in poverty and improved access to anthelminthic drugs among high-risk groups. These estimates were calculated using disability weights (factors that quantify the magnitude of health loss associated with specific health outcomes) based on symptomatic infection, wasting and mild abdominopelvic problems, with no attributed mortality. Recently, girls and women of reproductive age have been included 
as a high-risk group for anthelminthic treatment programmes, on the basis in part of the epidemiological links between $T$. trichiura infection and risk of anaemia in this group ${ }^{264}$. Trichuriasis is most common among those living in tropical regions in conditions of extreme poverty (that is, on less than US $\$ 1.90$ per day). Many of the factors that feed extreme poverty are linked to the risk of T. trichiura infection, which in turn contributes to the underlying causes of poverty. Thus, effective control of T. trichiura infection should contribute to reductions in poverty through improvements in health, educational achievement and economic productivity.

\section{Ascariasis}

As for trichuriasis, the burden of ascariasis is associated with the chronic and insidious effects that this disease has on the health and quality of life of infected individuals. A. lumbricoides, like T. trichiura, has a substantial role in childhood protein energy malnutrition and reduced food intake, leading to growth retardation, poor cognitive development, school absenteeism and poor academic performance. Collectively, these effects reduce an individual's productivity, thereby limiting the economic prospects of regions where A. lumbricoides is endemic $^{265-267}$.

The unique hepatic migration of Ascaris spp. can contribute to liver inflammation. An extensive prospective study in hospital patients in India revealed that in $14.5 \%$ of patients with liver abscess the cause was biliary ascariasis, and 11 patients had intact Ascaris spp. larvae within the liver abscess ${ }^{107}$. High burdens of adult worms are associated with life-threatening complications in children and adults, generally caused by intestinal and biliary obstruction ${ }^{268}$ among those with worm burdens of $>60$ parasites $^{269}$. Airway obstruction, a potential life-threatening event arising from $A$. lumbricoides infection, has also been reported ${ }^{270-272}$; however, this condition is rare, and there are no available data on its prevalence. The global DALY estimate for ascariasis was 0.861 million in $2017\left(\right.$ REF $\left.^{263}\right)$. Compared with data published in 2007, ascariasis was associated with the largest decrease in DALYs among all intestinal nematode infections, possibly owing to deworming programmes and socio-economic development. Ascariasis has been associated with more-severe asthma symptoms among children living in urban areas of the tropics, an effect attributed to strong pro-allergic effects of low levels of exposure to the parasite ${ }^{273,274}$.

\section{Outlook}

\section{Drug treatment and parasitological monitoring}

Complex and multifaceted challenges remain in the elimination of Ascaris spp. and Trichuris spp. infections. These challenges include the sustainability of preventive chemotherapy; the choice of at-risk groups (for example, at present, adult men are excluded from MDA programmes, and a study in Myanmar identified that this group has substantial burdens of both hookworm and T. trichiura infections ${ }^{275}$ ); the possible emergence of anthelminthic drug resistance; and the fact that a pan-STH vaccine ${ }^{226}$ is an ambitious endeavour. Furthermore, the emerging data on the effect of WASH programmes ${ }^{208}$ suggest that while the prevalence of STH infection remains high, MDA will still be required, and the benefits of WASH programmes will only be realized in the longer term. Certainly, the funding of initiatives such as the DeWorm 3 project ${ }^{276}$ is a welcome endeavour that will test the feasibility of interrupting transmission of STH infections using biannual MDA targeting all age groups, coupled with large-scale application of PCR assays for monitoring the efficacy of drug treatment. An argument that is gaining momentum is the need to move away from focusing on the treatment of only school-age children to a community-wide approach, especially in high transmission areas ${ }^{277}$. A large-scale randomized trial in Kenya that compared three treatment strategies (the annual treatment of the whole community, the biannual treatment of the whole community and the current focus of annually treating only children of 2-14 years of age) concluded that annual or biannual community treatment was more effective in reducing the prevalence and intensity of hookworm infections than treatment of school-age children only, but this approach needs to be explored in the context of infections with Ascaris spp. and T. trichiura ${ }^{278}$.

We urgently require well-designed, long-term epidemiological studies providing quantitative data, including data for appropriate mathematical modelling, to plan future elimination strategies. In this context, parasitological monitoring ${ }^{279}$, combined with the development of appropriate mathematical modelling approaches, is a key component required to improve our understanding of the efficacy of control strategies. New methods need to be developed to enable rapid measurement of the prevalence of STH infections in preschool children, school-age children, women of reproductive age and other at-risk groups, providing a more complete picture of the burden of STH infections in the entire community. In this context, the most urgent need is for accurate estimates of key parameters that can be fitted to mathematical models to assess the effect of treatment in key at-risk groups; such parameters include density dependence in worm fecundity (which is observed as a reduction in egg production with increasing worm burdens), parasite life expectancy, egg survival in the environment and age-specific force of infection, which describes the per capita rate at which susceptible individuals acquire infection $^{280}$.

\section{The development of new drugs}

The long-term effectiveness of the drugs currently available to treat Ascaris spp. and Trichuris spp. infections is a major concern and underpins the need for novel drug discovery. Encouragingly, drugs with new mechanisms of action and new drug candidates from natural products are being discovered. Further, access to the genomes of these ${ }^{82,281}$ and many other parasites ${ }^{282}$ offers the prospect of enhanced target-based screening for new anthelminthics. A chemogenomics approach (which takes the most promising drug targets in parasite genomes and explores their potential) is underway. For Trichuris spp., 40 priority targets were associated with 720 drug-like compounds (181 of which reached phase III/IV clinical trials for other human therapeutic applications and, therefore, would 


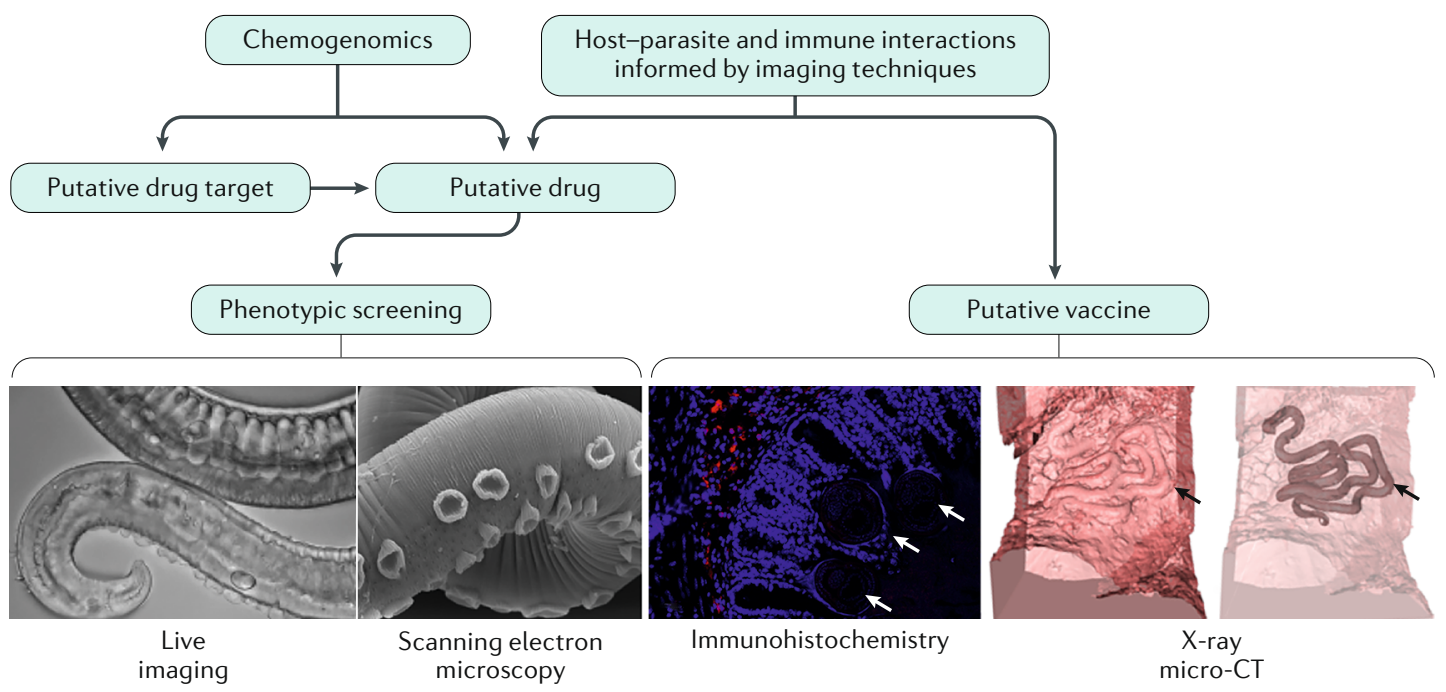

Fig. 6 | Outlook for the development of novel drugs for soil-transmitted helminth infections. Chemogenomics approaches will help to identify new candidate anthelminthic drugs targeting Ascaris spp. and Trichuris spp. Targets common to all soil-transmitted helminths (STHs) will be of particular interest. Advanced, automated phenotypic screening platforms of putative new drugs will emerge. An improved understanding of the worm life cycle, host-parasite interactions and host immunity to infection, informed by imaging techniques, may assist in adding context to omics-based discoveries, and, as a result, additional candidate targets might be identified, as well as challenges in developing new therapies. The live imaging photo shows the front end of an adult Trichuris muris worm. The scanning electron microscopy image shows the front end of an adult T. muris worm, highlighting structures called cuticular inflations ${ }^{313}$. The immunohistochemistry image shows neutrophils (red) in the lamina propria of the colon around an adult T. muris worm (arrows). The X-ray micro-CT images show 3D rendering of the host intestinal mucosa (pink). The positioning of adult T. muris within the epithelial cell layer (black arrows) is made clear by altering transparency of the rendering. Images courtesy of J. O'Sullivan and H. Smith, University of Manchester, Manchester, UK.

probably have good host tolerance ${ }^{282}$ ). For Ascaris spp., new targets with their variety of inhibitors may also offer new routes to drug discovery ${ }^{281}$.

Phenotypic screening, using live, ex vivo nematodes, has resulted in the discovery of most currently available anthelminthics ${ }^{283}$ and is likely to remain an important approach in the future. New platforms encompassing automated phenotyping that are suited to high-throughput chemical screening for motility and growth impairment in C. elegans and parasitic nematodes are available ${ }^{284}$. Such platforms facilitate the testing of putative drugs across different parasite species, with the goal of discovering moieties with activity against trematodes and nematodes. The wealth of behavioural data on mutants of $C$. elegans is also an accessible resource in the search for new candidate drug targets ${ }^{285}$. Compounds active against parasites and C. elegans will facilitate genetic approaches to target identification. By this means, new classes of compounds with anthelminthic properties are emerging ${ }^{286}$, including some with activity against both adult and egg stages, which may interrupt transmission ${ }^{287}$. This feature could be important, as both Trichuris spp. and Ascaris spp. eggs can remain viable in soil for extended periods ${ }^{288}$.

The use of advanced imaging technologies may improve our understanding of parasite-host biology and facilitate the development of novel drugs against STHs in general (FIG. 6). One such example is X-ray CT, which provides re-constructed $3 \mathrm{D}$ images of parasites in situ and over time ${ }^{52}$. This technique can highlight in detail parasite interactions with host tissue. For example, the attachment site of T. trichiura, the epithelial tunnel, remains poorly understood. To date the tunnel has only been viewed by scanning electron microscopy ${ }^{289}$ of the surface of the epithelial cells that line the gut lumen and by conventional histology, which provides a $2 \mathrm{D}$ view $^{290}$. $3 \mathrm{D}$ imaging offers the potential to view the attachment site in a more-holistic way and has already begun to show the complexity of $T$. muris interactions with intestinal cells. This complex interplay may present particular challenges for worm clearance ${ }^{52}$. Further, acknowledging and addressing important differences in the biology of Ascaris spp. and Trichuris spp. will facilitate the development of bespoke strategies to reduce prevalence and control morbidity. Future investigations of mechanisms of resistance and pathophysiology in animal models must focus on more physiologically relevant dosing regimens (low-dose infection and repeated low-dose ('trickle') infections) ${ }^{291}$. Mechanistic studies in mouse models must take into account the importance of context if we are to better model human trichuriasis and ascariasis. Such adjustments should include a consideration of the array of intrinsic and extrinsic host factors, such as genetics, age, sex, microbiota (which includes viruses, fungi and gut protozoa), co-infections, nutrition and reproductive state. Complex environmental factors affect individual variation in the immune status, and this interaction can be modelled, for example, in wild mouse populations ${ }^{292}$ and semi-wild systems ${ }^{293}$.

Anthelminthic drug resistance mechanisms may involve pharmacokinetics, drug detoxification and target-site modifications, all of which can shorten the 
useful lifespan of valuable compounds. Hence, discovering ways to circumvent resistance will be important in the future. Arguably the few compounds in repeated current use may increase the chances of resistance developing $^{294}$. Increasing the pipeline of new compounds will be important, as well as rotating or combining drug treatments. Resistance may be under-reported if we only screen for known resistance-associated polymorphisms. Improved molecular markers ${ }^{295}$ are needed to better understand resistance, especially when planning large-scale deworming programmes worldwide.

\section{Targeting liver immunity}

Stimulating host immunity may offer a therapeutic avenue, and there is emerging evidence for the role of the liver in immunity to ascariasis ${ }^{116,296}$. The liver proteome has been investigated in two inbred mouse strains, susceptible and resistant to Ascaris spp. infection ${ }^{119}$. Higher levels of mitochondrial proteins involved in oxidative phosphorylation were observed in the resistant strain (both at baseline and during infection) than in the susceptible strain. Thus, an intrinsic difference in the levels of reactive oxygen species in the liver could provide an advantage against the parasite in the resistant strain ${ }^{121}$. In another study, a lower burden of Ascaris spp. larvae was observed in the lungs of reinfected mice than in the lungs of mice infected for the first time. Further, lesions caused by hepatocyte necrosis and infiltration of eosinophils and neutrophils were more pronounced in the reinfected group; thus, the more pronounced hepatic immune response in the reinfected group resulted in a reduced lung larval burden ${ }^{44}$. Novel therapies targeting the liver could conceivably stop larval migration, reducing tissue damage and impairing development of adult worms.

\section{The development of vaccines}

Concerns remain that MDA alone will not be sufficient to eliminate STH infections, owing to rapid reinfection in environments where long-lived eggs survive, poor drug efficacy particularly against $T$. trichiura, the possibility of drug resistance and a lack of access to clean water and adequate sanitation. Thus, vaccination will be a continued focus for the future. However, in contrast to the efforts made to develop an anti-hookworm vaccine, progress in anti-Ascaris spp. and anti-Trichuris spp. vaccines has been slow. Pigs exposed to a vaccine composed of UV-irradiated, and thereby attenuated, A. suum eggs demonstrated enhanced immunity to a challenge with infective eggs, evidenced by reduced numbers of migrating larvae and adult worms in the intestine ${ }^{297}$. However, exposure to a mixture of antigens carries the risk of inducing allergic responses due to the allergenic properties of these antigens. The genes encoding several chemically defined antigens have been cloned, the recombinant proteins have been expressed, and six antigens have been targeted for further investigation ${ }^{226}$, including As14 (an antigen found in both larval and adult Ascaris spp. worms that induces a $64 \%$ reduction in the number of larvae after vaccination in mice ${ }^{298}$ ), As16 (REF. ${ }^{299}$ ) and As37 (REF. $\left.{ }^{300}\right)$. T. muris has not been studied as extensively as A. suum with respect to the development of recombinant antigens $^{226}$. Antigens derived from the stichosome have induced substantial reductions in worm burdens in a mouse model ${ }^{225}$. Further, the T. muris whey acidic protein (rTm-WAP49), secreted from the parasite's stichosome and with tentatively ascribed pore-forming activity, has been proposed as a promising vaccine candidate ${ }^{301}$, suggesting that the evaluation of T. muris recombinant proteins as immunogenic entities is gathering pace. rTm-WAP49 achieved a $48 \%$ reduction in worm burden in mice and showed high sequence conservation with the T. trichiura WAP proteins ${ }^{301}$. More recently, a novel $\mathrm{CD} 4^{+} \mathrm{T}$ cell epitope-based vaccine has also been shown to promote protective immunity in the mouse model ${ }^{302}$.

\section{Final words}

STHs are complex pathogens, and their control presents complex challenges, which differ according to context. Thus, formulating control guidelines and approaches that are universally applicable is a non-trivial task. A comprehensive approach including MDA, education and sanitation is crucial, in parallel with basic biological research. Enabling regions to take ownership of control programmes, thereby moving towards self-sustainability in both drug administration and drug procurement, is a key goal. In this context, new targets and indicators have been set by the $\mathrm{WHO}^{210}$. For example, the number of regions supporting deworming programmes with domestic funds is scheduled to increase from 5 in 2023 to 25 in 2030. Further, improved sanitation is a major goal, with the ambitious target of decreasing open defaecation to $0 \%$ in STH-endemic areas by 2030 , with progress towards this goal monitored from national surveys and census data. In addition to enabling regions to take ownership of control programmes, building a critical mass of scientists conducting infectious disease research in their home regions, where STH infections are endemic, is equally important. Multiple unmet needs exist in the field of basic biology of infection, including the need to develop affordable, sensitive diagnostic tools to monitor parasite prevalence and infection intensity and innovation in vaccine research. Nevertheless, the current pace of technological advances in biological research combined with the growth of multidisciplinary approaches suggests that living with STH infections will one day be the exception rather than the rule in regions where STHs are now endemic.

Published online: 28 May 2020
1. Araujo, A., Reinhard, K. J., Ferreira, L. F. \& Gardner, S. L. Parasites as probes for prehistoric human migrations? Trends Parasitol. 24, 112-115 (2008).

2. Hawash, M. B. et al. Whipworms in humans and pigs: origins and demography. Parasit. Vectors 9, 37 (2016).

3. Ledger, M. et al. Parasite infection at the early farming community of Çatalhöyük. Antiquity 93, 573-587 (2019).

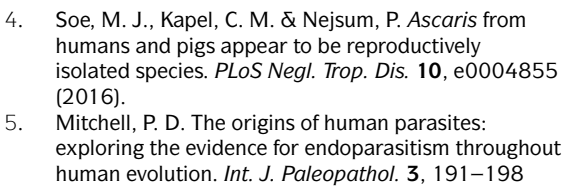

4. Soe, M. J., Kapel, C. M. \& Nejsum, P. Ascaris from humans and pigs appear to be reproductively isolated species. PLoS Negl. Trop. Dis. 10, e0004855 (2016).

5. Mitchell, P. D. The origins of human parasites: exploring the evidence for endoparasitism throughout human evolution. Int. J. Paleopathol. 3, 191-198 (2013).
6. Pullan, R. L., Smith, J. L., Jasrasaria, R. \& Brooker, S. J. Global numbers of infection and disease burden of soil transmitted helminth infections in 2010. Parasites Vectors 7, 37 (2014)

7. Hotez, P. J. et al. The global burden of disease study 2010: interpretation and implications for the neglected tropical diseases. PLoS Negl. Trop. Dis. 8, e2865 (2014). 
8. World Health Organization. Preventive chemotherapy to control soil-transmitted helminths in at-risk population groups: guideline (WHO, 2017)

9. Hotez, P. J. Global deworming: moving past albendazole and mebendazole. Lancet Infect. Dis. 17, 1101-1102 (2017)

10. GBD 2015 DALYs and HALE Collaborators. Global, regional, and national disability-adjusted life-years (DALYs) for 315 diseases and injuries and healthy life expectancy (HALE), 1990-2015: a systematic analysis for the Global Burden of Disease Study 2015. Lancet 388, 1603-1658 (2016).

11. World Health Organization. Eliminating soil-transmitted helminthiases as a public health problem in children: progress report 2001-2010 and strategic plan 2011-2020 (WHO, 2012).

12. Yakob, L. et al. Slaving and release in co-infection control Parasites Vectors 6,157 (2013).

13. Pullan, R. \& Brooker, S. The health impact of polyparasitism in humans: are we under-estimating the burden of parasitic diseases? Parasitology 135 783-794 (2008)

14. Brooker, S., Clements, A. C. \& Bundy, D. A. Global epidemiology, ecology and control of soil-transmitted helminth infections. Adv. Parasitol. 62, 221-261 (2006).

15. Owada, K. et al. Spatial distribution and populations at risk of $A$. lumbricoides and T. trichiura co-infections and infection intensity classes: an ecological study. Parasites Vectors 11, 535 (2018)

16. Global Health Data Exchange. GBD results tool. GHDx http://ghdx.healthdata.org/gbd-results-tool (2020).

17. Beer, R. J. The relationship between Trichuris trichiura (Linnaeus 1758) of man and Trichuris suis (Schrank 1788) of the pig. Res. Vet. Sci. 20, 47-54 (1976).

18. Bundy, D. A. \& Cooper, E. S. Trichuris and trichuriasis in humans. Adv. Parasitol. 28, 107-173 (1989).

19. Summers, R. W., Elliott, D. E., Urban, J. F. Jr., Thompson, R. A. \& Weinstock, J. V. Trichuris suis therapy for active ulcerative colitis: a randomized controlled trial. Gastroenterology 128, 825-832 (2005).

20. Williams, A. R. et al. Immune responses and parasitological observations induced during probiotic treatment with medicinal Trichuris suis ova in a healthy volunteer. Immunol. Lett. 188, 32-37 (2017).

21. Ghai, R. R. et al. Hidden population structure and cross-species transmission of whipworms (Trichuris sp.) in humans and non-human primates in Uganda. PLoS Negl. Trop. Dis. 8, e3256 (2014).

22. Bundy, D. A., Cooper, E. S., Thompson, D. E. Didier, J. M. \& Simmons, I. Epidemiology and population dynamics of Ascaris lumbricoides and Trichuris trichiura infection in the same community. Trans. R. Soc. Tropical Med. Hyg. 81, 987-993 (1987).

23. Bundy, D. A., Cooper, E. S., Thompson, D. E., Anderson, R. M. \& Didier, J. M. Age-related prevalence and intensity of Trichuris trichiura infection in a St. Lucian community. Trans. R. Soc. Tropical Med. Hyg. 81, 85-94 (1987)

24. de Silva, N. R. et al. Soil-transmitted helminth infections: updating the global picture. Trends Parasitol. 19, 547-551 (2003)

25. Schulz, J. D., Moser, W., Hurlimann, E. \& Keiser, J. Preventive chemotherapy in the fight against soil-transmitted helminthiasis: achievements and limitations. Trends Parasitol. 34, 590-602 (2018).

26. Elliott, D. E. in Sleisenger and Fordtran's Gastrintestinal and Liver Disease: Pathophysiology Diagnosis/Management (eds Friedman, L. S., Feldman, M., Brandt, L. J.) 2435-2457 (Elsevier Philadelphia, 2006).

27. Wright, J. E., Werkman, M., Dunn, J. C. \& Anderson, R. M. Current epidemiological evidence for predisposition to high or low intensity human helminth infection: a systematic review. Parasites Vectors 11, 65 (2018)

28. Bundy, D. A. et al. Predisposition to Trichuris trichiura infection in humans. Epidemiol. Infect. 98, 65-71 (1987).

29. Ellis, M. K et al. Familial aggregation of human susceptibility to co- and multiple helminth infections in a population from the Poyang Lake region, China. Int. J. Parasitol. 37, 1153-1161 (2007)

30. Williams-Blangero, S. et al. Two quantitative trait loci influence whipworm (Trichuris trichiura) infection in a Nepalese population. J. Infect. Dis. 197, 1198-1203 (2008).

This study identified two quantitative trait loci that influence egg counts for Trichuris trichiura infection, one on chromosome 9 and one on chromosome 12
31. Costa, R. D. et al. Effect of polymorphisms on TGFB on allergic asthma and helminth infection in an African admixed population. Ann. Allergy Asthma Immunol. 118, 483-488.e1 (2017).

32. Global Burden of Disease Study 2013 Collaborators. Global, regional, and national incidence, prevalence, and years lived with disability for 301 acute and chronic diseases and injuries in 188 countries, 1990-2013: a systematic analysis for the Global Burden of Disease Study 2013. Lancet 386 , 743-800 (2015)

33. Avery, R. H., Wall, L. A., Verhoeve, V. I., Gipson, K. S. $\&$ Malone, J. B. Molecular confirmation of Ascaris suum: further investigation into the zoonotic origin of infection in an 8-year-old boy with Loeffler syndrome. Vector Borne Zoonotic Dis. 18, 638-640 (2018)

34. Sadaow, L. et al. Molecular identification of Ascaris lumbricoides and Ascaris suum recovered from humans and pigs in Thailand, Lao PDR, and Myanmar Parasitol. Res. 117, 2427-2436 (2018).

35. Monteiro, K. J. L. et al. Genetic diversity of Ascaris spp. infecting humans and pigs in distinct Brazilian regions, as revealed by mitochondrial DNA. PLoS One 14, e0218867 (2019).

36. Betson, M., Nejsum, P., Bendall, R. P., Deb, R. M $\&$ Stothard, J. R. Molecular epidemiology of ascariasis: a global perspective on the transmission dynamics of Ascaris in people and pigs. J. Infect. Dis. 210, 932-941 (2014).

37. Nejsum, P., Betson, M., Bendall, R. P., Thamsborg, S. M. $\&$ Stothard, J. R. Assessing the zoonotic potential of Ascaris suum and Trichuris suis: looking to the future from an analysis of the past. J. Helminthol. 86, 148-155 (2012)

38. Benjamin-Chung, J. et al. The interaction of deworming, improved sanitation, and household flooring with soil-transmitted helminth infection in rural Bangladesh. PLoS Negl. Trop. Dis. 9, e0004256 (2015).

39. Kightlinger, L. K., Seed, J. R. \& Kightlinger, M. B. Ascaris lumbricoides intensity in relation to environmental, socioeconomic, and behavioral determinants of exposure to infection in children from southeast Madagascar. J. Parasitol. 84, 480-484 (1998).

40. Croll, N. A. \& Ghadirian, E. Wormy persons: contributions to the nature and patterns of overdispersion with Ascaris lumbricoides, Ancylosotma duodenale, Necator americanus and Trichuris trichiura. Tropical Geographical Med. 33, 241-248 (1981).

41. Holland, C. V. et al. Intestinal helminthiases in relation to the socioeconomic environment of Panamanian children. Soc. Sci. Med. 26, 209-213 (1988).

42. Haswell-Elkins, M., Elkins, D. \& Anderson, R. M. The influence of individual, social group and household factors on the distribution of Ascaris lumbricoides within a community and implications for control strategies. Parasitology 98, 125-134 (1989).

43. Holland, C. V. et al. The epidemiology of Ascaris lumbricoides and other soil-transmitted helminths in primary school children from lle-lfe, Nigeria. Parasitology 99, 275-285 (1989).

44. Nogueira, D. S. et al. Multiple exposures to Ascaris suum induce tissue injury and mixed Th2/Th 17 immune response in mice. PLoS Negl. Trop. Dis. 10 e0004382 (2016)

45. Chan, L., Bundy, D. A. \& Kan, S. P. Genetic relatedness as a determinant of predisposition to Ascaris lumbricoides and Trichuris trichiura infection. Parasitology 108, 77-80 (1994)

46. Williams-Blangero, $\mathrm{S}$. et al. Genetic analysis of susceptibility to infection with Ascaris lumbricoides. Am. J. Tropical Med. Hyg. 60, 921-926 (1999).

47. Williams-Blangero, $\mathrm{S}$. et al. Genes on chromosomes 1 and 13 have significant effects on Ascaris infection. Proc. Natl Acad. Sci. USA 99, 5533-5538 (2002).

48. Peisong, G. et al. An asthma-associated genetic variant of STAT6 predicts low burden of Ascaris worm infestation. Genes. Immun. 5, 58-62 (2004).

49. Williams-Blangero, S. et al. Localization of multiple quantitative trait loci influencing susceptibility to infection with Ascaris lumbricoides. J. Infect. Dis. 197 66-71 (2008)

50. Acevedo, N. et al. Association between total immunoglobulin $\mathrm{E}$ and antibody responses to naturally acquired Ascaris lumbricoides infection and polymorphisms of immune system-related LIG4, TNFSF13B and IRS2 genes. Clin. Exp. Immunol. 157 282-290 (2009).

51. Dold, C. \& Holland, C. V. Investigating the underlying mechanism of resistance to Ascaris infection. Microbes Infect. 13, 624-631 (2011).
52. O'Sullivan, J. D. B. et al. X-ray micro-computed tomography (muCT): an emerging opportunity in parasite imaging. Parasitology 145, 848-854 (2018).

53. Starborg, T. et al. Experimental steering of electron microscopy studies using prior X-ray computed tomography. Ultramicroscopy 201, 58-67 (2019).

54. Hayes, K. S. et al. Exploitation of the intestinal microflora by the parasitic nematode Trichuris muris. Science 328, 1391-1394 (2010).

The study demonstrated a crucial relationship between intestinal bacteria and Trichuris muris in the mouse, with successful establishment of infection dependent on the microbiota.

55. Cliffe, L. J. \& Grencis, R. K. The Trichuris muris system a paradigm of resistance and susceptibility to intestinal nematode infection. Adv. Parasitol. 57 255-307 (2004)

56. Hurst, R. J. \& Else, K. J. Trichuris muris research revisited: a journey through time. Parasitology 140 , 1325-1339 (2013).

57. Pike, E. H. Egg output of Trichuris muris (Schrank, 1788). J. Parasitol. 55, 1046-1049 (1969).

58. Eichenberger, R. M. et al. Characterization of Trichuris muris secreted proteins and extracellular vesicles provides new insights into host-parasite communication J. Extracell. Vesicles 7, 1428004 (2018)

59. Bancroft, A. J. et al. The major secreted protein of the whipworm parasite tethers to matrix and inhibits interleukin-13 function. Nat. Commun. 10, 2344 (2019).

60. Leroux, L. P. et al. Analysis of the Trichuris suis excretory/secretory proteins as a function of life cycle stage and their immunomodulatory properties. Sci. Rep. 8, 15921 (2018)

61. Faulkner, H. et al. Age- and infection intensitydependent cytokine and antibody production in human trichuriasis: the importance of IgE. J. Infect. Dis. 185, 665-672 (2002).

62. de Ruiter, K. et al. Helminth infections drive heterogeneity in human type 2 and regulatory cells. Sci. Transl Med. 12, eaaw3703 (2020).

63. Turner, J. D. et al. Th2 cytokines are associated with reduced worm burdens in a human intestinal helminth infection. J. Infect. Dis. 188, 1768-1775 (2003). The authors report that an increase in type 2 immunity-associated cytokines correlates with an age-dependent reduction in Ascaris lumbricoides infection intensity

64. Dige, A. et al. Mucosal and systemic immune modulation by Trichuris trichiura in a self-infected individual. Parasite Immunol. 39, e12394 (2017).

65. Broadhurst, M. J. et al. IL-22+ CD4+ T cells are associated with therapeutic Trichuris trichiura infection in an ulcerative colitis patient. Sci. Trans/ Med. 2, 60 ra88 (2010).

This study showed that Trichuris trichiura infection may decrease the symptoms of colitis by promoting type 2 immunity dependent goblet cell hyperplasia and mucus production

66. Kringel, H., Iburg, T., Dawson, H., Aasted, B. \& Roepstorff, A. A time course study of immunological responses in Trichuris suis infected pigs demonstrates induction of a local type 2 response associated with worm burden. Int. J. Parasitol. 36, 915-924 (2006).

67. Else, K. J., Finkelman, F. D., Maliszewski, C. R. \& Grencis, R. K. Cytokine-mediated regulation of chronic intestinal helminth infection. J. Exp. Med. 179, 347-351 (1994)

68. Grencis, R. K. Immunity to helminths: resistance, regulation, and susceptibility to gastrointestinal nematodes. Annu. Rev. Immunol. 33, 201-225 (2015).

69. Hadidi, S. et al. Myeloid cell-specific expression of Ship 1 regulates IL-12 production and immunity to helminth infection. Mucosal Immunol. 5, 535-543 (2012).

70. Artis, D New weapons in the war on worms: identification of putative mechanisms of immunemediated expulsion of gastrointestinal nematodes. Int. J. Parasitol. 36, 723-733 (2006).

71. Klementowicz, J. E., Travis, M. A. \& Grencis, R. K. Trichuris muris: a model of gastrointestinal parasite infection. Semin. Immunopathol. 34, 815-828 (2012).

72. Sorobetea, D., Svensson-Frej, M. \& Grencis, R. Immunity to gastrointestinal nematode infections. Mucosal Immunol. 11, 304-315 (2018).

73. Blackwell, N. M. \& Else, K. J. B cells and antibodies are required for resistance to the parasitic gastrointestinal nematode Trichuris muris. Infect. Immun. 69 3860-3868 (2001). 
74. Perrigoue, J. G. et al. MHC class II-dependent basophil-CD4+ T cell interactions promote $\mathrm{T}(\mathrm{H}) 2$ cytokine-dependent immunity. Nat. Immunol. 10 697-705 (2009)

75. Huang, X., Zeng, L. R., Chen, F. S., Zhu, J. P. \& Zhu, M. H. Trichuris suis ova therapy in inflammatory bowel disease: a meta-analysis. Medicine 97, e12087 (2018).

76. Bager, P. Use of Trichuris suis ova (TSO) therapy for the treatment of allergy. Arb. Paul Ehrlich Inst. Bundesinstitut Impfstoffe Biomed. Arzneim. Langen Hess. 97, 128-129 (2013).

77. Schölmerich, J. et al. A randomised, double-blind, placebo-controlled trial of Trichuris suis ova in active Crohn's disease. J. Crohns Colitis 11, 390-399 (2017).

78. Sobotkova, K. et al. Helminth therapy - from the parasite perspective. Trends Parasitol. 35, 501-515 (2019).

79. Bager, P. et al. Trichuris suis ova therapy for allergic rhinitis: a randomized, double-blind, placebocontrolled clinical trial. J. Allergy Clin. Immunol. 125 123-130.e1-3 (2010).

80. Hasnain, S. Z. et al. Muc5ac: a critical component mediating the rejection of enteric nematodes. J. Exp. Med. 208, 893-900 (2011).

81. Hasnain, S. Z. et al. Mucin gene deficiency in mice impairs host resistance to an enteric parasitic infection. Gastroenterology 138, 1763-1771 (2010). This study provided evidence of a functional role for mucins in type 2 controlled anti-nematode immunity using the mouse model of human trichuriasis.

82. Foth, B. J. et al. Whipworm genome and dual-species transcriptome analyses provide molecular insights into an intimate host-parasite interaction. Nat. Genet. 46 693-700 (2014)

83. Khan, W. I. et al. Modulation of intestinal muscle contraction by interleukin-9 (IL-9) or IL-9 neutralization: correlation with worm expulsion in murine nematode infections. Infect. Immun. $\mathbf{7 1}$ 2430-2438 (2003).

84. Cliffe, L. J. et al. Accelerated intestinal epithelial cell turnover: a new mechanism of parasite expulsion. Science 308, 1463-1465 (2005).

85. Jarrett, E. E. \& Miller, H. R. Production and activities of IgE in helminth infection. Prog. Allergy 31 178-233 (1982)

86. Else, K. J. \& Grencis, R. K. Antibody-independent effector mechanisms in resistance to the intestinal nematode parasite Trichuris muris. Infect. Immun. 64 2950-2954 (1996)

87. Constant, S., Schweitzer, N., West, J., Ranney, P. $\&$ Bottomly, K. B lymphocytes can be competent antigen-presenting cells for priming CD4+ T cells to protein antigens in vivo. J. Immunol. 155, 3734-3741 (1995)

88. Lund, F. E., Garvy, B. A., Randall, T. D. \& Harris, D. P. Regulatory roles for cytokine-producing B cells in infection and autoimmune disease. Curr. Dir. Autoimmun. 8, 25-54 (2005).

89. Wojciechowski, W. et al. Cytokine-producing effector B cells regulate type 2 immunity to $H$. polygyrus. Immunity 30, 421-433 (2009).

90. Schopf, L. R., Hoffmann, K. F., Cheever, A. W. Urban, J. F. Jr. \& Wynn, T. A. IL-10 is critical for host resistance and survival during gastrointestinal helminth infection. J. Immunol. 168, 2383-2392 (2002).

91. Duque-Correa, M. A. et al. Exclusive dependence of IL-10Ra signalling on intestinal microbiota homeostasis and control of whipworm infection. PLoS Pathog. 15, e1007265 (2019).

92. Houlden, A. et al. Chronic Trichuris muris infection in c57bl/6 mice causes significant changes in host microbiota and metabolome: effects reversed by pathogen clearance. PLoS One 10, e0125945 (2015).

93. Holm, J. B. et al. Chronic Trichuris muris infection decreases diversity of the intestinal microbiota and concomitantly increases the abundance of lactobacilli. PLoS One 10, e0125495 (2015)

94. Li, R. W. et al. Alterations in the porcine colon microbiota induced by the gastrointestinal nematode Trichuris suis. Infect. Immun. 80, 2150-2157 (2012).

95. Wu, S. et al. Worm burden-dependent disruption of the porcine colon microbiota by Trichuris suis infection. PLoS One 7, e35470 (2012).

96. Lee, S. C. et al. Helminth colonization is associated with increased diversity of the gut microbiota. PLoS Negl. Trop. Dis. 8, e2880 (2014).

97. Cooper, P. et al. Patent human infections with the whipworm, Trichuris trichiura, are not associated with alterations in the faecal microbiota. PLoS One $\mathbf{8}$, e76573 (2013).

98. White, E. C. et al. Manipulation of host and parasite microbiotas: survival strategies during chronic nematode infection. Sci. Adv. 4, eaap7399 (2018).

99. Stephenson, L. S. \& Holland, C. The Impact of Helminth Infections on Human Nutrition: schistosomes and soil-transmitted helminths 93 (Taylor and Francis, 1987)

100. Wong, M. S. \& Bundy, D. A. Quantitative assessment of contamination of soil by the eggs of Ascaris lumbricoides and Trichuris trichiura. Trans. R. Soc Tropical Med. Hyg. 84, 567-570 (1990).

101. Douvres, F. W. \& Urban, J. F. Jr. Factors contributing to the in vitro development of Ascaris suum from second-stage larvae to mature adults. J. Parasitol. 69 549-558 (1983)

102. Fagerholm, H. P., Nansen, P., Roepstorff, A., Frandsen, F. \& Eriksen, L. Differentiation of cuticular structures during the growth of the third-stage larva of Ascaris suum (Nematoda, Ascaridoidea) after emerging from the egg. J. Parasitol. 86, 421-427 (2000).

103. Pilitt, P. A., Lichtenfels, J. R., Tromba, F. G $\&$ Madden, P. A. Differentiation of late fourth and early fifth stages of Ascaris suum Goeze, 1782 (Nematoda: Ascaridoidea) in swine. Proc. Helminthol. Soc. 48, 1-7 (1981)

104. Anderson, R. M. in The Population Dynamics of Infectious Diseases: Theory and Applications (ed. Anderson, R. M.) 67-108 (Springer, 1982).

105. Pawlowski, Z. S. \& Arfaa, F. in Tropical and Geographical Medicine (eds Warren, K. S. \& Mahmoud, A. A. F. 347-358 (McGraw-Hill, 1984).

106. Read, A. F. \& Skorping, A. The evolution of tissue migration by parasitic nematode larvae. Parasitology 111, 359-371 (1995).

107. Javid, G. et al. Ascaris-induced liver abscess. World J. Surg. 23, 1191-1194 (1999).

108. Ribeiro, J. D. F. G. Eosinophilic lung diseases. Paediatr. Respir. Rev. 3, 278-284 (2002)

This review highlights eosinophilic lung diseases, with an emphasis on lung pathology due to parasitic infections including Ascaris lumbricoides infection.

109. Hagel, I. et al. Ascaris reinfection of slum children: relation with the IgE response. Clin. Exp. Immunol. 94, 80-83 (1993)

110. Palmer, D. R., Hall, A., Haque, R. \& Anwar, K. S Antibody isotype responses to antigens of Ascaris lumbricoides in a case-control study of persistently heavily infected Bangladeshi children. Parasitology 111, 385-393 (1995).

111. McSharry, C., Xia, Y., Holland, C. V. \& Kennedy, M. W. Natural immunity to Ascaris lumbricoides associated with immunoglobulin E antibody to ABA-1 allergen and inflammation indicators in children. Infect. Immun. 67, 484-489 (1999)

112. King, E. M. et al. Immuno-epidemiology of Ascaris lumbricoides infection in a high transmission community: antibody responses and their impact on current and future infection intensity. Parasite Immunol. 27, 89-96 (2005).

113. Cooper, P. J. et al. Human infection with Ascaris lumbricoides is associated with a polarized cytokine response. J. Infect. Dis. 182, 1207-1213 (2000).

114. Jackson, J. A. et al. T helper cell type 2 responsiveness predicts future susceptibility to gastrointestinal nematodes in humans. J. Infect. Dis. 190, 1804-1811 (2004).

115. Cooper, P. J. et al. Repeated treatments with albendazole enhance Th2 responses to Ascaris lumbricoides, but not to aeroallergens, in children from rural communities in the tropics. J. Infect. Dis. 198, 1237-1242 (2008)

This paper presents data indicating that repeated doses of albendazole over the course of 1 year are associated with enhanced production of Ascaris lumbricoides-specific type 2 immunity cytokines in humans.

116. Holland, C. V., Behnke, J.M. and Dold, C. in Ascaris the Neglected Parasite (ed. Holland, C. V.) 107-125 (Elsevier, 2013).

117. Lewis, R., Behnke, J. M., Stafford, P. \& Holland, C. V. Dose-dependent impact of larval Ascaris suum on hos body weight in the mouse model. J. Helminthol. $\mathbf{8 3}$ 1-5 (2009).

118. Deslyper, G., Holland, C. V., Colgan, T. J. $\&$ Carolan, J. C. The liver proteome in a mouse model for Ascaris suum resistance and susceptibility: evidence for an altered innate immune response. Parasites Vectors 12, 402 (2019).
119. Lewis, R., Behnke, J. M., Stafford, P. \& Holland, C. V. The development of a mouse model to explore resistance and susceptibility to early Ascaris suum infection. Parasitology 132, 289-300 (2006).

120. Dold, C., Cassidy, J. P., Stafford, P., Behnke, J. M $\&$ Holland, C. V. Genetic influence on the kinetics and associated pathology of the early stage (intestinalhepatic) migration of Ascaris suum in mice. Parasitology 137, 173-185 (2010).

121. Deslyper, G., Colgan, T. J., Cooper, A. J., Holland, C. V. $\&$ Carolan, J. C. A proteomic investigation of hepatic resistance to Ascaris in a murine model. PLoS Negl. Trop. Dis. 10, e0004837 (2016).

122. Gazzinelli-Guimaraes, P. H. et al. Parasitological and immunological aspects of early Ascaris spp. infection in mice. Int. J. Parasitol. 43, 697-706 (2013).

123. Weatherhead, J. E. et al. Ascaris larval infection and lung invasion directly induce severe allergic airway disease in mice. Infect. Immun. 86, e00533-18 (2018).

124. Gazzinelli-Guimaraes, P. H. et al. Allergen presensitization drives an eosinophil-dependent arrest in lung-specific helminth development. J. Clin. Invest'. 130, 3686-3701 (2019)

125. Guo, L. et al. Innate immunological function of $\mathrm{TH} 2$ cells in vivo. Nat. Immunol. 16, 1051-1059 (2015)

126. Dowling, D. J. et al. Ascaris lumbricoides pseudocoelomic body fluid induces a partially activated dendritic cell phenotype with Th2 promoting ability in vivo. Int. J. Parasitol. 41, 255-261 (2011).

127. Favoretto, B. C. et al. High molecular weight components containing $\mathrm{N}$-linked oligosaccharides of Ascaris suum extract inhibit the dendritic cells activation through DC-SIGN and MR. Mol. Immunol. 87, 33-46 (2017)

128. Almeida, S., Nejsum, P. \& Williams, A. R. Modulation of human macrophage activity by Ascaris antigens is dependent on macrophage polarization state. Immunobiology 223, 405-412 (2018).

129. Titz, T. O. et al. Ascaris suum infection modulates inflammation: implication of CD4(+) CD25(high) Foxp3 (+) T cells and IL-10. Parasite Immunol. 39 , e12453 (2017).

130. Nascimento, W. C. et al. Immunomodulation of liver injury by Ascaris suum extract in an experimental model of autoimmune hepatitis. Parasitol. Res. 113 3309-3317 (2014).

131. Paterson, J. C., Garside, P., Kennedy, M. W. \& Lawrence, C. E. Modulation of a heterologous immune response by the products of Ascaris suum. Infect. Immun. 70, 6058-6067 (2002).

132. Gazzinelli-Guimaraes, P. H. et al. Concomitant helminth infection downmodulates the Vaccinia virus-specific immune response and potentiates virus-associated pathology. Int. J. Parasitol. 47, 1-10 (2017).

133. Suzuki, M. et al. Presensitization to Ascaris antigens promotes induction of mite-specific IgE upon mite antigen inhalation in mice. Allergol. Int. 65, 44-51 (2016).

134. Jungersen, G., Fagerholm, H. P., Nansen, P. \& Eriksen, L. Development of patent Ascaris suum infections in pigs following intravenous administration of larvae hatched in vitro. Parasitology 119, 503-508 (1999).

135. Masure, D. et al. The intestinal expulsion of the roundworm Ascaris suum is associated with eosinophils, intra-epithelial T cells and decreased intestinal transit time. PLoS Negl. Trop. Dis. 7, e2588 (2013).

136. Schwartz, B. A. J. Ascaris larvae as a cause of liver and lung lesions in swine. J. Parasitol. 19, 17-24 (1932).

137. Copeman, D. B. \& Gaafar, S. M. Sequential development of hepatic lesions of ascaridosis in colostrum-deprived pigs. Aust. Vet. J. 48, 263-268 (1972)

138. Perez, J., Garcia, P. M., Mozos, E., Bautista, M. J. \& Carrasco, L. Immunohistochemical characterization of hepatic lesions associated with migrating larvae of Ascaris suum in pigs. J. Comp. Pathol. 124, 200-206 (2001).

139. Masure, D. et al. A role for eosinophils in the intestinal immunity against infective Ascaris suum larvae. PLoS Negl. Trop. Dis. 7, e2138 (2013).

140. Ashraf, M., Urban, J. F. Jr., Lee, T. D. \& Lee, C. M Characterization of isolated porcine intestinal mucosal mast cells following infection with Ascaris suum. Vet. Parasitol. 29, 143-158 (1988).

141. Uston, P. I., Urban, J. F. Jr., Ashraf, M., Lee, C. M. \& Ampy, F. R. L3L4ES antigen and secretagogues induce histamine release from porcine peripheral blood basophils after Ascaris suum infection. Parasitol. Res. $100,603-611$ (2007). 
142. Urban, J. F. Jr., Alizadeh, H. \& Romanowski, R. D. Ascaris suum: development of intestinal immunity to infective second-stage larvae in swine. Exp. Parasitol. 66, 66-77 (1988).

143. Wang, Y. et al. Ascaris suum infection was associated with a worm-independent reduction in microbia diversity and altered metabolic potential in the porcine gut microbiome. Int. J. Parasitol. 49, 247-256 (2019).

144. McCraw, B. M. \& Greenway, J. A. Ascaris suum infection in calves. 3. Pathology. Can. J. Comp. Med. 34, 247-255 (1970).

145. Fallis, A. M. Ascaris lumbricoides infection in guinea pigs with special reference to eosinophilia and resistance. Can. J. Res. 26, 307-327 (1948)

146. Arean, V. M. \& Crandall, C. A. The effect of immunization on the fate of injected second stage Ascaris lumbricoides larvae in the rabbit. Am. J. Tropical Med. Hyg. 11, 369-379 (1962).

147. Cho, S. et al. Migration behaviour and pathogenesis of five ascarid nematode species in the Mongolian gerbil Meriones unguiculatus. J. Helminthol. 81, 43-47 (2007).

148. Weiszer, I., Patterson, R. \& Pruzansky, J. J. Ascaris hypersensitivity in the rhesus monkey: I. A model fo the study of immediate type hypersensitity in the primate. J. Allergy 41, 14-22 (1968).

149. Pritchard, D. I. et al. Laboratory infection of primates with Ascaris suum to provide a model of allergic bronchoconstriction. Clin. Exp. Immunol. 54 469-476 (1983)

150. Patterson, R., Harris, K. E. \& Pruzansky, J. J. Induction of IgE-mediated cutaneous, cellular, and airway reactivity in rhesus monkeys by Ascaris suum infection. J. Lab. Clin. Med. 101, 864-872 (1983).

151. Layrisse, M., Aparcedo, L., Martinez-Torres, C. \& Roche, M. Blood loss due to infection with Trichuris trichiura. Am. J. Trop. Med. Hyg. 16, 613-619 (1967).

152. Ramdath, D. D., Simeon, D. T., Wong, M. S. \& Grantham-McGregor, S. M. Iron status of schoolchildren with varying intensities of Trichuris trichiura infection. Parasitology 110, 347-351 (1995).

153. Robertson, L. J., Crompton, D. W., Sanjur, D. \& Nesheim, M. C. Haemoglobin concentrations and concomitant infections of hookworm and Trichuris trichiura in Panamanian primary schoolchildren. Trans. R. Soc. Trop. Med. Hyg. 86, 654-656 (1992).

154. Gyorkos, T. W., Gilbert, N. L., Larocque, R. \& Casapia, M Trichuris and hookworm infections associated with anaemia during pregnancy. Trop. Med. Int. Health 16 531-537 (2011).

155. Cooper, E. S. \& Bundy, D. A. Trichuris is not trivial Parasitol. Today 4, 301-306 (1988).

156. Wolfe, M. S. Oxyuris, Trichostrongylus and Trichuris. Clin Gastroenterol. 7, 201-217 (1978).

157. Khuroo, M. S., Khuroo, M. S. \& Khuroo, N. S. Trichuris dysentery syndrome: a common cause of chronic iron deficiency anemia in adults in an endemic area (with videos). Gastrointest. Endoscopy 71, 200-204 (2010).

158. Stephenson, L. S., Holland, C. V. \& Cooper, E. S. The public health significance of Trichuris trichiura. Parasitology 121, S73-S95 (2000)

159. Kaminsky RG, C. R., \& Flores, C. A. Growth retardation and severe anemia in children with Trichuris dysenteric syndrome. Asian Pac. J. Trop. Biomed. 5, 591-597 (2015).

160. Al-Mekhlafi, M. H. et al. Anaemia and iron deficiency anaemia among aboriginal schoolchildren in rural Peninsular Malaysia: an update on a continuing problem. Trans. R. Soc. Tropical Med. Hyg. 102 1046-1052 (2008)

161. Sarkar, M., Mahesh, D. M. \& Madabhavi, I. Digital clubbing. Lung India 29, 354-362 (2012)

162. Cooper, E. S., Bundy, D. A. \& Henry, F. J. Chronic dysentery, stunting, and whipworm infestation. Lancet 2, 280-281 (1986)

163. MacDonald, T. T. et al. Immunoepidemiology of intestinal helminthic infections. 3. Mucosal macrophages and cytokine production in the colon of children with Trichuris trichiura dysentery. Trans. $R$. Soc. Tropical Med. Hyg. 88, 265-268 (1994).

164. Ok, K. S. et al. Trichuris trichiura infection diagnosed by colonoscopy: case reports and review of literature. Korean J. Parasitol. 47, 275-280 (2009).

165. Jha, A. K., Goenka, M. K. \& Suchismita, A. Clinical correlates of trichuriasis diagnosed at colonoscopy. Indian. J. Gastroenterol. 36, 420-423 (2017).

166. Martin, I. et al. Dynamic changes in human-gut microbiome in relation to a placebo-controlled anthelminthic trial in Indonesia. PLoS Negl. Trop. Dis. 12, e0006620 (2018).

167. Gilman, R. H. et al. The adverse consequences of heavy Trichuris infection. Trans. R. Soc. Tropical Med. Hyg. 77, 432-438 (1983).

168. Shin, J. L., Gardiner, G. W., Deitel, W. \& Kandel, C. Does whipworm increase the pathogenicity of Campylobacter jejuni? A clinical correlate of an experimental observation. Can. J. Gastroenterol. 18, 175-177 (2004)

169. Jensen, L. A., Marlin, J. W., Dyck, D. D. \& Laubach, H. E. Prevalence of multi-gastrointestinal infections with helminth, protozoan and Campylobacter spp. in Guatemalan children. J. Infect. Dev. Ctries. 3, 229-234 (2009).

170. Mansfield, L. S. et al. Enhancement of disease and pathology by synergy of Trichuris suis and Campylobacter jejuni in the colon of immunologically naive swine. Am. J. Tropical Med. Hyg. 68, 70-80 (2003).

171. Croll, N. A., Anderson, R. M., Gyorkos, T. W. \& Ghadirian, E. The population biology and control of Ascaris lumbricoides in a rural community in Iran. Trans. R. Soc. Tropical Med. Hyg. 76, 187-197 (1982).

172. Thein, H., Than, S., Htay Htay, A., Myint, L. $\&$ Thein Maung, M. Epidemiology and transmission dynamics of Ascaris lumbricoides in Okpo village, rural Burma. Trans. R. Soc. Tropical Med. Hyg. 78, 497-504 (1984)

173. Löffler, W. Transient lung infiltrations with blood eosinophilia. Int. Arch. Allergy Appl. Immunol. 8, 54-59 (1956).

174. Khuroo, N. S., Khuroo, M. S. \& Khuroo, M. S. Gastric ascariasis presenting as unique dyspeptic symptoms in an endemic area. Am. J. Gastroenterol. 105, 1675-1677 (2010).

175. Das, A. K. Hepatic and biliary ascariasis. J. Glob. Infect. Dis. 6, 65-72 (2014)

176. Khuroo, M. S. Ascariasis. Gastroenterol. Clin. North. Am. 25, 553-577 (1996).

177. Efem, S. E. Ascaris lumbricoides and intestinal perforation. Br. J. Surg. 74, 643-644 (1987).

178. Paul, M. The movements of the adult Ascaris lumbricoides. Br. J. Surg. 59, 437-442 (1972).

179. Villamizar, E., Mendez, M., Bonilla, E., Varon, H. $\&$ de Onatra, S. Ascaris lumbricoides infestation as a cause of intestinal obstruction in children: experience with 87 cases. J. Pediatric Surg. 31, 201-204 (1996).

180. Nokes, C. \& Bundy, D. A. Does helminth infection affect mental processing and educational achievement? Parasitol. Today 10, 14-18 (1994). This article reviews the evidence linking helminth infections with impaired cognitive function and the difficulties associated with such studies.

181. Symons, L. E. Anorexia: occurrence, pathophysiology, and possible causes in parasitic infections. Adv. Parasitol. 24, 103-133 (1985).

182. Stephenson, L. S. The contribution of Ascaris limbricoides to malnutrition in children. Parasitology 81, 221-233 (1980)

183. Katz, N., Chaves, A. \& Pellegrino, J. A simple device for quantitative stool thick-smear technique in Schistosomiasis mansoni. Rev. Inst. Med. Trop. Sao Paulo 14, 397-400 (1972).

184. World Health Organization. Prevention and control of schistosomiasis and soil-transmitted helminthiasis: report of a WHO expert committee (WHO, 2002).

185. Nikolay, B., Brooker, S. J. \& Pullan, R. L. Sensitivity of diagnostic tests for human soil-transmitted helminth infections: a meta-analysis in the absence of a true gold standard. Int. J. Parasitol. 44, 765-774 (2014)

186. Bogoch, I. I. et al. Mobile phone microscopy for the diagnosis of soil-transmitted helminth infections: a proof-of-concept study. Am. J. Tropical Med. Hyg. $\mathbf{8 8}$ 626-629 (2013)

This paper explores the use of mobile phone microscopy as a novel method for point-of-care diagnosis of soil-transmitted helminth infections

187. Moser, W. et al. Diagnostic comparison between FECPAKC2 and the Kato-Katz method for analyzing soil-transmitted helminth eggs in stool. PLoS Neglected Tropical Dis. 12, e0006562 (2018)

188. Arndt, M. B. et al. Impact of helminth diagnostic test performance on estimation of risk factors and outcomes in HIV-positive adults. PLoS One 8, e81915 (2013).

189. Easton, A. V. et al. Multi-parallel qPCR provides increased sensitivity and diagnostic breadth for gastrointestinal parasites of humans: field-based inferences on the impact of mass deworming. Parasites Vectors 9,38 (2016).
190. Liu, J. et al. A laboratory-developed TaqMan Array Card for simultaneous detection of 19 enteropathogens. J. Clin. Microbiol. 51, 472-480 (2013).

191. Mationg, M. L. S. et al. Status of soil-transmitted helminth infections in schoolchildren in Laguna Province, the Philippines: determined by parasitological and molecular diagnostic techniques. PLoS Negl. Trop. Dis. 11, e0006022 (2017).

192. O'Connell, E. M. \& Nutman, T. B. Molecular diagnostics for soil-transmitted helminths. Am. J. Tropical Med. Hyg. 95, 508-513 (2016)

193. Phuphisut, O. et al. Triplex polymerase chain reaction assay for detection of major soil-transmitted helminths, Ascaris lumbricoides, Trichuris trichiura Necator americanus, in fecal samples. Southeast. Asian J. Tropical Med. Public. Health 45, 267-275 (2014).

194. Llewellyn, S. et al. Application of a multiplex quantitative PCR to assess prevalence and intensity of intestinal parasite infections in a controlled clinical trial. PLoS Negl. Trop. Dis. 10, e0004380 (2016).

195. Cunningham, L. J. et al. Developing a real-time PCR assay based on multiplex high-resolution melt-curve analysis: a pilot study in detection and discrimination of soil-transmitted helminth and schistosome species. Parasitology 145, 1733-1738 (2018).

196. Mejia, R. et al. A novel, multi-parallel, real-time polymerase chain reaction approach for eight gastrointestinal parasites provides improved diagnostic capabilities to resource-limited at-risk populations. Am. J. Tropical Med. Hyg. 88 1041-1047 (2013).

197. Pilotte, N. et al. Improved PCR-based detection of soil transmitted helminth infections using a nextgeneration sequencing approach to assay design. PLoS Negl. Trop. Dis. 10, e0004578 (2016).

198. Rashwan, N. Diawara, A., Scott, M. E. \& Prichard, R. K Isothermal diagnostic assays for the detection of soiltransmitted helminths based on the SmartAmp2 method. Parasites Vectors 10, 496 (2017).

199. Schrader, C., Schielke, A., Ellerbroek, L. \& Johne, R. PCR inhibitors-occurrence, properties and removal. J. Appl. Microbiol. 113, 1014-1026 (2012).

200. World Health Organization. Prevention and control of intestinal parasitic infections: report of a WHO exper committee (WHO, 1987).

201. Marocco, C., Bangert, M., Joseph, S. A., Fitzpatrick, C. $\&$ Montresor, A. Preventive chemotherapy in one year reduces by over $80 \%$ the number of individuals with soil-transmitted helminthiases causing morbidity: results from meta-analysis. Trans. $R$. Soc. Tropical Med. Hyg. 111, 12-17 (2017)

202. Bah, Y M et al Soil-transmitted helminth infection in school age children in Sierra Leone after a decade of preventive chemotherapy interventions. Infect. Dis. Poverty 8, 41 (2019).

203. Shumbej, T. et al. Impact of annual preventive mass chemotherapy for soil-transmitted helminths among primary school children in an endemic area of Gurage zone: a prospective cross-sectional study. Res. Rep. Tropical Med. 10, 109-118 (2019).

204. Bartram, J. \& Cairncross, S. Hygiene, sanitation, and water: forgotten foundations of health. PLoS Med. 7 . e1000367 (2010).

205. Fewtrell, L. et al. Water, sanitation, and hygiene interventions to reduce diarrhoea in less developed countries: a systematic review and meta-analysis. Lancet. Infect. Dis. 5, 42-52 (2005).

This article provides a systematic review and metaanalysis summarizing the importance of water, sanitation and hygiene (WASH) interventions in the control of soil transmitted helminth infections

206. Cairncross, S. et al. Water, sanitation and hygiene for the prevention of diarrhoea. Int. J. Epidemiol. 39 (Suppl 1), i193-i205 (2010)

207. Strunz, E. C. et al. Water, sanitation, hygiene, and soiltransmitted helminth infection: a systematic review and meta-analysis. PLoS Med 11, e1001620 (2014).

208. Vaz Nery, S. et al. The role of water, sanitation and hygiene interventions in reducing soil-transmitted helminths: interpreting the evidence and identifying next steps. Parasites Vectors 12, 273 (2019).

209. Freeman, M. C. et al. Challenges and opportunities for control and elimination of soil-transmitted helminth infection beyond 2020. PLoS Negl Trop Dis 13 e0007201 (2019)

210. World Health Organization. 2030 targets for soiltransmitted helminthiases control programmes (WHO, 2020).

211. Turner, K. J., Fisher, E. H. \& McWilliam, A. S. Homology between roundworm (Ascaris) and hookworm (N. americanus) antigens detected by human IgE 
antibodies. Australian J. Exp. Biol. Med. Sci. 58, 249-257 (1980).

212. Correa-Oliveira, R. et al. Human antibody responses against schistosomal antigens. I. Antibodies from patients with Ancylostoma, Ascaris lumbricoides or Schistosoma mansoni infections react with schistosome antigens. Am. J. Tropical Med. Hyg. 38, 348-355 (1988).

213. Betschart, B., Marti, S. \& Glaser, M. Antibodies against the cuticlin of Ascaris suum cross-react with epicuticular structures of filarial parasites. Acta Tropica 47, 331-338 (1990)

214. Cuellar, C., Fenoy, S. \& Guillen, J. L. Cross-reactions of sera from Toxascaris leonina and Ascaris suum infected mice with Toxocara canis, Toxascaris leonina and Ascaris suum antigens. Int. J. Parasitol. 25 731-739 (1995)

215. Santos, A. B. et al. Cross-reactive IgE antibody responses to tropomyosins from Ascaris lumbricoides and cockroach. J. Allergy Clin. Immunol. 121 1040-1046.e 1 (2008).

216. Caraballo, L. \& Acevedo, N. Allergy in the tropics: the impact of cross-reactivity between mites and Ascaris. Front. Biosci. 3, 51-64 (2011).

217. Elsemore, D. A. et al. Enzyme-linked immunosorbent assay for coproantigen detection of Trichuris vulpis in dogs. J. Veterinary Diagn. Invest. 26, 404-411 (2014).

218. Martinez-Perez, J. M., Vandekerckhove, E., Vlaminck, J., Geldhof, P. \& Martinez-Valladares, M. Serological detection of Ascaris suum at fattening pig farms is linked with performance and management indices. Veterinary Parasitol. 248, 33-38 (2017).

219. Geng, J., Elsemore, D. A., Oudin, N. \& Ketzis, J. K Diagnosis of feline whipworm infection using a coproantigen ELISA and the prevalence in feral cats in southern Florida. Veterinary Parasitol. Regional Stud. Rep. 14, 181-186 (2018).

220. Lassen, B. et al. Anti-Ascaris suum IgG antibodies in fattening pigs with different respiratory conditions. Veterinary Parasitol. 265, 85-90 (2019).

221. Yoshida, A., Kikuchi, T., Nakagaki, S. \& Maruyama, H. Optimal ELISA antigen for the diagnosis of Ascaris suum infection in humans. Parasitology Res. 115 4701-4705 (2016)

222. Lopes, C. A. et al. Anti-Ascaris suum immunoglobulin $Y$ as a novel biotechnological tool for the diagnosis of human ascariasis. J. Helminthol. 94, e71 (2019).

223. Gazzinelli-Guimaraes, A. C. et al. IgG induced by vaccination with Ascaris suum extracts is protective against infection. Front. Immunol. 9, 2535 (2018).

224. Robinson, K., Bellaby, T. \& Wakelin, D. Efficacy of oral vaccination against the murine intestinal parasite Trichuris muris is dependent upon host genetics. Infect. Immun. 63, 1762-1766 (1995).

225. Dixon, H., Little, M. C. \& Else, K. J. Characterisation of the protective immune response following subcutaneous vaccination of susceptible mice against Trichuris muris. Int. J. Parasitol. 40, 683-693 (2010).

226. Zhan, B. et al. Advancing a multivalent 'Pan-anthelmintic' vaccine against soil-transmitted nematode infections. Expert. Rev. Vaccines 13, 321-331 (2014).

227. World Health Organization. Preventive chemotherapy in human helminthiasis. Coordinated use of anthelminthic drugs in control interventions: a manual for health professionals and programme managers (WHO, 2006).

228. Olliaro, P. et al. Potential drug development candidates for human soil-transmitted helminthiases. PLoS Negl. Trop. Dis. 5, e1138 (2011)

229. Palmeirim, M. S., Ame, S. M., Ali, S. M., Hattendorf, J. $\&$ Keiser, J. Efficacy and safety of a single dose versus a multiple dose regimen of mebendazole against hookworm infections in children: a randomised, double-blind trial. EClinicalMedicine 1, 7-13 (2018)

230. World Health Organization. WHO model list of essentia medicines (WHO, 2017)

231. Moser, W., Schindler, C. \& Keiser, J. in Highlighting Operational and Implementation Research for Contro of Helminthiasis (ed. Keiser, J.) 91-115 (Academic Press, 2019)

232. Palmeirim, M. S. et al. Efficacy and safety of coadministered ivermectin plus albendazole for treating soil-transmitted helminths: a systematic review, meta-analysis and individual patient data analysis. PLoS Negl. Trop. Dis. 12, e0006458 (2018).

233. Patel, C. et al. Efficacy and safety of ivermectin and albendazole co-administration in school-aged children and adults infected with Trichuris trichiura: study protocol for a multi-country randomized controlled double-blind trial. BMC Infect. Dis. 19, 262 (2019).

234. Opoku, N. O. et al. Single dose moxidectin versus ivermectin for Onchocerca volvulus infection in Ghana,
Liberia, and the Democratic Republic of the Congo: a randomised, controlled, double-blind phase 3 trial. Lancet 392, 1207-1216 (2018).

235. Keller, L. et al. Efficacy and safety of ascending dosages of moxidectin and moxidectin-albendazole against Trichuris trichiura in adolescents: a randomized controlled trial. Clin. Infect. Dis. 70, 1193-1201 (2020).

236. Wimmersberger, D. et al. Efficacy and safety of ivermectin against Trichuris trichiura in preschooland school-aged children: a randomized controlled dose-finding trial. Clin. Infect. Dis. 67, 1247-1255 (2018).

237. Kopp, S. \& Keiser, J. in Kucers' the Use of Antibiotics: a Clinical Review of Antibacterial, Antifungal, Antiparasitic, and Antiviral Drugs, 7th edn (ed. Grayson, M. L. et al.) 3381-3384 (CRC Press, 2017).

238. Moser, W. et al. Efficacy and tolerability of triple drug therapy with albendazole, pyrantel pamoate, and oxantel pamoate compared with albendazole plus oxantel pamoate, pyrantel pamoate plus oxantel pamoate, and mebendazole plus pyrantel pamoate and oxantel pamoate against hookworm infections in school-aged children in Laos: a randomised, single-blind trial. Lancet. Infect. Dis. 18, 729-737 (2018).

239. Krucken, J. et al. Anthelmintic cyclcooctadepsipeptides: complex in structure and mode of action. Trends Parasitol. 28, 385-394 (2012).

240. Karpstein, T. et al. Evaluation of emodepside in laboratory models of human intestinal nematode and schistosome infections. Parasites Vectors 12, 226 (2019).

241. Matamoros, G. et al. High endemicity of soiltransmitted helminths in a population frequently exposed to albendazole but no evidence of antiparasitic resistance. Trop. Med. Infect. Dis. 4, 73 (2019).

242. Moser, W., Schindler, C. \& Keiser, J. Efficacy of recommended drugs against soil transmitted helminths: systematic review and network meta-analysis. $B M J$ 358, j4307 (2017).

243. Khuroo, M. S., Rather, A. A. Khuroo, N. S $\&$ Khuroo, M. S. Hepatobiliary and pancreatic ascariasis. World J. Gastroenterol. 22, 7507-7517 (2016).

244. Alhamid, A. et al. Successful elimination of gallbladder ascariasis by conservative therapy, followed by cholecystectomy due to developing cholecystitis Case Rep. Gastrointest. Med. 2018, 5831257 (2018)

245. Gupta, S. et al. Ascaris lumbricoides: an unusual aetiology of gastric perforation. J. Surg. Case Rep. 2012, rjs008 (2012).

246. Campbell, S. J. et al. Complexities and perplexities: a critical appraisal of the evidence for soil-transmitted helminth infection-related morbidity. PLoS Negl. Trop. Dis. 10, e0004566 (2016)

247. Taylor-Robinson, D. C., Maayan, N., Donegan, S., Chaplin, M. \& Garner, P. Public health deworming programmes for soil-transmitted helminths in children living in endemic areas. Cochrane Database Syst. Rev. 9, CD000371 (2019).

248. Welch, V. A. et al. Mass deworming to improve developmental health and wellbeing of children in low-income and middle-income countries: a systematic review and network meta-analysis. Lancet. Glob. Health 5, e40-e50 (2017).

249. Taylor-Robinson, D. C., Maayan, N., Soares-Weiser, K., Donegan, S. \& Garner, P. Deworming drugs for soiltransmitted intestinal worms in children: effects on nutritional indicators, haemoglobin, and school performance. Cochrane Database of Syst. Rev. 7 , CD000371 (2015).

250. Pabalan, N. et al. Soil-transmitted helminth infection, loss of education and cognitive impairment in schoolaged children: a systematic review and meta-analysis. PLoS Negl. Trop. Dis. 12, e0005523 (2018)

251. Yap, P., Utzinger, J., Hattendorf, J. \& Steinmann, P Influence of nutrition on infection and re-infection with soil-transmitted helminths: a systematic review. Parasites Vectors 7, 229 (2014).

252. Cooper, E. S., Duff, E. M., Howell, S. \& Bundy, D. A. 'Catch-up' growth velocities after treatment for Trichuris dysentery syndrome. Trans. R. Soc. Tropical Med. Hyg. 89, 653 (1995).

253. Cooper, E. S., Bundy, D. A., MacDonald, T. T. \& Golden, M. H. Growth suppression in the Trichuris dysentery syndrome. Eur. J. Clin. Nutr. 44, 285-291 (1990).

254. GBD 2017 Disease and Injury Incidence and Prevalence Collaborators. Global, regional, and national incidence, prevalence, and years lived with disability for 354 diseases and injuries for 195 countries and territories, 1990-2017: a systematic analysis for the Global Burden of Disease Study 2017. Lancet 392, 1789-1858 (2018)

255. The World Bank. Decline of global extreme poverty continues but has slowed: World Bank. The World Bank https://www.worldbank.org/en/news/ press-release/2018/09/19/decline-of-globalextreme-poverty-continues-but-has-slowed-world-bank (2018).

256. Brooker, S. Estimating the global distribution and disease burden of intestinal nematode infections: adding up the numbers-a review. Int. J. Parasitol. 40 1137-1144 (2010)

257. Gardner, J. M., Grantham-McGregor, S. \& Baddeley, A Trichuris trichiura infection and cognitive function in Jamaican school children. Ann. Tropical Med. Parasitol. 90, 55-63 (1996)

258. Simeon, D. T., Grantham-McGregor, S. M. Callender, J. E. \& Wong, M. S. Treatment of Trichuris trichiura infections improves growth, spelling scores and school attendance in some children. J. Nutr. 125, 1875-1883 (1995)

259. Ahmed, A. et al. Soil-transmitted helminthiasis: a critical but neglected factor influencing school participation of Aboriginal children in rural Malaysia. Parasitology 139, 802-808 (2012).

260. Callender, J., Grantham-McGregor, S., Walker, S. $\&$ Cooper, E. Developmental levels and nutritional status of children with the Trichuris dysentery syndrome. Trans. R. Soc. Tropical Med. Hyg. 87, 528-529 (1993).

261. Stephenson, L. S., Latham, M. C., Adams, E. J. Kinoti, S. N. \& Pertet, A. Physical fitness, growth and appetite of Kenyan school boys with hookworm, Trichuris trichiura and Ascaris lumbricoides infections are improved four months after a single dose of albendazole. J. Nutr. 123, 1036-1046 (1993).

262. Lenk, E. J., Redekop, W. K., Luyendijk, M., Rijnsburger, A. J. \& Severens, J. L. Productivity loss related to neglected tropical diseases eligible for preventive chemotherapy: a systematic literature review. PLoS Negl. Trop. Dis. 10, e0004397 (2016).

263. GBD 2017 DALYs and HALE Collaborators. Global, regional, and national disability-adjusted life-years (DALYs) for 359 diseases and injuries and healthy life expectancy (HALE) for 195 countries and territories, 1990-2017: a systematic analysis for the Global Burden of Disease Study 2017. Lancet 392 1859-1922 (2018).

264. Gyorkos, T. W. et al. The right to deworming: the case for girls and women of reproductive age. PLoS Negl. Trop. Dis. 12, e0006740 (2018).

265. Galgamuwa, L. S., Iddawela, D. \& Dharmaratne, S. D. Prevalence and intensity of Ascaris lumbricoides infections in relation to undernutrition among children in a tea plantation community, Sri Lanka: a crosssectional study. BMC Pediatrics 18, 13 (2018).

266. Drake, L. J., Jukes, M. C. H., Sternberg, R. J. $\&$ Bundy, D. A. P. Geohelminth infections (ascariasis, trichuriasis and hookworm): cognitive and developmental impacts. Semin. Pediatric Infect. Dis. 11, 245-251 (2000)

267. Dickson, R., Awasthi, S., Williamson, P., Demellweek, C. $\&$ Garner, P. Effects of treatment for intestinal helminth infection on growth and cognitive performance in children: systematic review of randomised trials. $B M J$ 320, 1697-1701 (2000).

268. O'Lorcain, P. \& Holland, C. V. The public health importance of Ascaris lumbricoides. Parasitology 121, S51-S71 (2000).

269. de Silva, N. R., Guyatt, H. L. \& Bundy, D. A. Morbidity and mortality due to Ascaris-induced intestinal obstruction. Trans. R. Soc. Tropical Med. Hyg. 91 31-36 (1997)

270. Roy, K., Kundra, P. \& Ravishankar, M. Unusual foreign body airway obstruction after laryngeal mask airway insertion. Anesthesia Analgesia 101, 294-295 (2005).

271. Husain, S. J., Zubairi, A. B., Sultan, N., Beg, M. A. \& Mehraj, V. Recurrent episodes of upper airway blockage associated with Ascaris lumbricoides causing cardiopulmonary arrest in a young patient. BMJ Case Rep. 2009, bcr0120091415 (2009).

272. Prakash, S., Sitalakshmi, N., Singh, J., Dayal, M. \& Gogia, A. R. Ascaris: an unusual cause of airway obstruction during general anesthesia with ProSeal laryngeal mask airway. J. Anaesthesiol. Clin. Pharmacol. 30, 298-300 (2014).

273. Endara, P. et al. Effect of urban vs. rural residence on the association between atopy and wheeze in Latin America: findings from a case-control analysis. Clin. Exp. Allergy 45, 438-447 (2015). 
274. Caraballo, L., Acevedo, N. \& Zakzuk, J. Ascariasis as a model to study the helminth/allergy relationships. Parasite Immunol. 41, e 12595 (2019).

275. Dunn, J. C. et al. A cross-sectional survey of soiltransmitted helminthiases in two Myanmar villages receiving mass drug administration: epidemiology of infection with a focus on adults. Parasites Vectors 10. 374 (2017).

276. Asbjornsdottir, K. H. et al. Assessing the feasibility of interrupting the transmission of soil-transmitted helminths through mass drug administration: the DeWorm3 cluster randomized trial protocol. PLoS Negl. Trop. Dis. 12, e0006166 (2018).

277. Anderson, R. M., Turner, H. C., Truscott, J. E., Hollingsworth, T. D. \& Brooker, S. J. Should the goal for the treatment of soil transmitted helminth (STH) infections be changed from morbidity control in children to community-wide transmission elimination? PLoS Negl. Trop. Dis. 9, e0003897 (2015).

278. Pullan, R. L. et al. Effects, equity, and cost of school-based and community-wide treatment strategies for soil-transmitted helminths in Kenya: a cluster-randomised controlled trial. Lancet 393 2039-2050 (2019)

279. Becker, S. L. et al. Toward the 2020 goal of soiltransmitted helminthiasis control and elimination. PLoS Negl. Trop. Dis. 12, e0006606 (2018).

280. Hollingsworth, T. D., Truscott, J.E. \& Anderson, R.M in Ascaris: the Neglected Parasite (ed. Holland, C.) 231-262 (Elsevier, 2013).

281. Jex, A. R. et al. Ascaris suum draft genome. Nature 479, 529-533 (2011).

282. Coghlan, A. et al. Comparative genomics of the major parasitic worms. Nat. Genet. 51, 163-174 (2019).

283. Kaminsky, R. et al. A new class of anthelmintics effective against drug-resistant nematodes. Nature 452, 176-180 (2008)

284. Partridge, F. A. et al. An automated high-throughput system for phenotypic screening of chemical libraries on C. elegans and parasitic nematodes. Int. $J$. Parasitol. Drugs Drug. Resistance 8, 8-21 (2018)

285. Yemini, E., Jucikas, T., Grundy, L. J., Brown, A. E. \& Schafer, W. R. A database of Caenorhabditis elegans behavioral phenotypes. Nat. Methods 10, 877-879 (2013).

286. Partridge, F. A. et al. Dihydrobenz[e][1,4]oxazepin $2(3 \mathrm{H})$-ones, a new anthelmintic chemotype immobilising whipworm and reducing infectivity in vivo. PLoS Negl. Trop. Dis. 11, e0005359 (2017).

287. Partridge, F. A. et al. 2,4-Diaminothieno[3,2-d] pyrimidines, a new class of anthelmintic with activity against adult and egg stages of whipworm. PLoS Negl. Trop. Dis. 12, e0006487 (2018).

This paper reports the discovery of a nove anthelminthic compound with activity against both adult Trichuris spp. parasites and the parasitic egg stage and discusses the potential for environmental control to break the parasite life cycle.

288. Mejer, H. \& Roepstorff, A. in Proc. 23rd International Conference of the World Association for the Advancement of Veterinary Parasitology 113 (International Conference of the World Association for the Advancement of Veterinary Parasitology, 2011).

289. Tilney, L. G., Connelly, P. S., Guild, G. M., Vranich, K. A $\&$ Artis, D. Adaptation of a nematode parasite to living within the mammalian epithelium. J. Exp. Zool. Part. A, Comp. Exp. Biol. 303, 927-945 (2005).

290. Lee, T. D. \& Wright, K. A. The morphology of the attachment and probable feeding site of the nematode Trichuris muris (Schrank, 1788) Hall, 1916. Can. J. Zool. 56, 1889-1905 (1978).

291. Glover, M., Colombo, S. A. P., Thornton, D. J. $\&$ Grencis, R. K. Trickle infection and immunity to Trichuris muris. PLoS Pathog. 15, e1007926 (2019).
292. Abolins, S. et al. The comparative immunology of wild and laboratory mice, Mus musculus domesticus. Nat. Commun. 8, 14811 (2017).

293. Leung, J. M. et al. Rapid environmental effects on gut nematode susceptibility in rewilded mice. PLoS Biol. 16, e2004108 (2018)

294. Vercruysse, J. et al. Is anthelmintic resistance a concern for the control of human soil-transmitted helminths? Int. J. Parasitol. Drugs Drug Resistance 1, 14-27 (2011)

295. Diawara, A. et al. Association between response to albendazole treatment and $\beta$-tubulin genotype frequencies in soil-transmitted helminths. PLoS Negl. Trop. Dis. 7, e2247 (2013).

296. Ronéus, O. Studies on the aetiology and pathogenesis of white spots in the liver of pigs. Acta Veterinaria Scandinavica 7 (Suppl 16), 1-112 (1966).

297. Urban, J. F. Jr. \& Tromba, F. G. An ultraviolet-attenuated egg vaccine for swine ascariasis: parameters affecting the development of protective immunity. Am. J. Veterinary Res. 45, 2104-2108 (1984).

298. Tsuji, N. et al. Intranasal immunization with recombinant Ascaris suum 14-kilodalton antigen coupled with cholera toxin B subunit induces protective immunity to $A$. suum infection in mice. Infect. Immun. 69, 7285-7292 (2001).

299. Wei, J. et al. Yeast-expressed recombinant As 16 protects mice against Ascaris suum infection through induction of a Th2-skewed immune response. PLOS Negl. Trop. Dis. 11, e0005769 (2017).

300. Versteeg, L. et al. Protective immunity elicited by the nematode-conserved As37 recombinant protein against Ascaris suum infection. PLoS Negl. Trop. Dis. 14, e0008057 (2020).

301. Briggs, N. et al. Trichuris muris whey acidic protein induces type 2 protective immunity against whipworm PLoS Pathog. 14, e1007273 (2018). The authors identify the Trichuris muris whey acidic protein as a promising human vaccine candidate.

302. Zawawi, A. et al. In silico design of a T-cell epitope vaccine candidate for parasitic helminth infection. PLoS Pathog. 16, e1008243 (2020).

303. Khurana, S. \& Sethi, S. Laboratory diagnosis of soil transmitted helminthiasis. Tropical Parasitol. 7 86-91 (2017).

304. Lamberton, P. H. \& Jourdan, P. M. Human ascariasis: diagnostics update. Curr. Tropical Med. Rep. 2 , 189-200 (2015)

305. Knopp, S. et al. A single FLOTAC is more sensitive than triplicate Kato-Katz for the diagnosis of low-intensity soil-transmitted helminth infections. Trans. R. Soc. Tropical Med. Hyg. 103, 347-354 (2009).

306. Cools, P. et al. Diagnostic performance of a single and duplicate Kato-Katz, Mini-FLOTAC, FECPAKG2 and qPCR for the detection and quantification of soiltransmitted helminths in three endemic countries. PLoS Negl. Trop. Dis. 13, e0007446 (2019).

307. Levecke, B. et al. A comparison of the sensitivity and fecal egg counts of the McMaster egg counting and Kato-Katz thick smear methods for soil-transmitte helminths. PLoS Negl. Trop. Dis. 5, e1201 (2011).

308. Shiraho, E. A. et al. Development of a loop mediated isothermal amplification for diagnosis of Ascaris lumbricoides in fecal samples. J. Parasitol. Res. 2016 7376207 (2016)

309. Holt, D. C. et al. Soil-transmitted helminths in children in a remote aboriginal community in the Northern Territory: hookworm is rare but Strongyloides stercoralis and Trichuris trichiura persist. Trop. Med. Infect. Dis. 2, 51 (2017).

310. Briolat, E. Worming it out. MRC http://www.bpod.mrc ac.uk/archive/2016/9/13 (2018).

311. Arnold, M., Alves, J. \& Plessis, J. in $A B C$ of Pediatric Surgical Imaging (eds Andronikou, S., Alexander, A.,
Kilborn, T., Millar, A. J. W. \& Daneman, A.) (Springer, 2010)

312. Yetim, I. et al. Rare cause of intestinal obstruction, Ascaris lumbricoides infestation: two case reports. Cases J. 2, 7970 (2018).

313. O’Sullivan, J. D. B., Cruickshank, S. M., Starborg, T. Withers, P. J. \& Else, K. J. Characterisation of cuticular inflation development and composition in Trichuris muris using correlative X-ray computed tomography and electron microscopy. Sci. Rep. 10, 5846 (2020).

314. Mayer, J. U. et al. Different populations of CD11b(+) dendritic cells drive Th2 responses in the small intestine and colon. Nat. Commun. 8, 15820 (2017).

315. Luda, K. M. et al. IRF8 transcription-factor-dependent classical dendritic cells are essential for intestinal t cell homeostasis. Immunity 44, 860-874 (2016).

316. Gold, M. J., Antignano, F., Hughes, M. R., Zaph, C. \& McNagny, K. M. Dendritic-cell expression of Ship 1 regulates Th2 immunity to helminth infection in mice. Eur. J. Immunol. 46, 122-130 (2016).

317. Owyang, A. M. et al. Interleukin 25 regulates type 2 cytokine-dependent immunity and limits chronic inflammation in the gastrointestinal tract. J. Exp. Med. 203, 843-849 (2006).

318. Taylor, B. C. et al. TSLP regulates intestinal immunity and inflammation in mouse models of helminth infection and colitis. J. Exp. Med. 206, 655-667 (2009).

319. Hopwood, T. W. et al. The circadian regulator BMAL1 programmes responses to parasitic worm infection via a dendritic cell clock. Sci. Rep. 8, 3782 (2018). The authors provide evidence for an increased susceptibility of mice to Trichuris muris infection if the infection is given at night rather than day; they also show that this time of day dependency of the outcome of infection involves a molecular clock within the dendritic cells.

\section{Acknowledgements}

The authors thank R. Forman, J. O'Sullivan and H. Smith (Lydia Becker Institute for Immunology and Inflammation, Faculty of Biology, Medicine and Health, University of Manchester, Manchester Academic Health Science Centre, Manchester, UK) for providing images and design of Fig. 3 and Fig. 6. The authors also thank J. O'Sullivan for design of Fig. 4. The authors acknowledge the Engineering and Physical Science Research Council (EPSRC) for funding the Henry Moseley X-ray Imaging Facility through grants (EP/F007906/1, EP/F001452/1, EP/ I02249X, EP/F028431/1 and EP/M022498/1 and platform grant EP/M010619/1) within the Henry Royce Institute.

\section{Author contributions}

Introduction (K.J.E. and C.V.H.); Epidemiology (P.J.C., L.L.B., S.O.A. and O.A.S.); Mechanisms/pathophysiology (K.J.E., R.K.G., C.V.H., R.T.F. and L.L.B.); Diagnosis, screening and prevention (P.J.C., L.L.B., S.O.A., O.A.S. and R.T.F.); Management (J.K., S.O.A. and O.A.S.); Quality of Life (P.J.C., S.O.A. and O.A.S.); Outlook (D.B.S. and C.V.H.); Overview of Primer (K.J.E.).

\section{Competing interests}

All authors declare no competing interests.

\section{Peer review information}

Nature Reviews Disease Primers thanks A. Clements, A. Loukas, J. Urban and the other, anonymous, reviewer(s) for their contribution to the peer review of this work.

\section{Publisher's note}

Springer Nature remains neutral with regard to jurisdictional claims in published maps and institutional affiliations.

C) Springer Nature Limited 2020 